ARTICLE

\title{
Cell type-specific delivery of short interfering RNAs by dye-functionalised theranostic nanoparticles
}

Adrian T. Press ${ }^{1,2, *}$, Anja Traeger ${ }^{2,3, *}$, Christian Pietsch 2,3 , Alexander Mosig ${ }^{1,4}$, Michael Wagner ${ }^{2,3}$, Mark G. Clemens ${ }^{1,5}$, Nayla Jbeily ${ }^{1}$, Nicole Koch ${ }^{6}$, Michael Gottschaldt ${ }^{2,3}$, Nicolas Bézière ${ }^{7}$, Volodymyr Ermolayev ${ }^{7}$, Vasilis Ntziachristos ${ }^{7}$, Jürgen Popp ${ }^{1,2,8,9}$, Michael M. Kessels ${ }^{6}$, Britta Qualmann ${ }^{2,6}$, Ulrich S. Schubert $2,3, \uparrow \&$ Michael Bauer ${ }^{1,2,9, \dagger}$

Efficient delivery of short interfering RNAs reflects a prerequisite for the development of RNA interference therapeutics. Here, we describe highly specific nanoparticles, based on near infrared fluorescent polymethine dye-derived targeting moieties coupled to biodegradable polymers. The fluorescent dye, even when coupled to a nanoparticle, mimics a ligand for hepatic parenchymal uptake transporters resulting in hepatobiliary clearance of approximately $95 \%$ of the dye within 45 min. Body distribution, hepatocyte uptake and excretion into bile of the dye itself, or dye-coupled nanoparticles can be tracked by intravital microscopy or even non-invasively by multispectral optoacoustic tomography. Efficacy of delivery is demonstrated in vivo using 3-hydroxy-3-methyl-glutaryl-CoA reductase siRNA as an active payload resulting in a reduction of plasma cholesterol levels if siRNA was formulated into dye-functionalised nanoparticles. This suggests that organ-selective uptake of a near infrared dye can be efficiently transferred to theranostic nanoparticles allowing novel possibilities for personalised silencing of disease-associated genes.

\footnotetext{
${ }^{1}$ Center for Sepsis Control \& Care (CSCC), Jena University Hospital, Erlanger Allee 101, 07747 Jena, Germany. ${ }^{2}$ Jena Center for Soft Matter (JCSM), Friedrich Schiller University Jena, Phiolophenweg 7, 07743 Jena, Germany. ${ }^{3}$ Laboratory of Organic and Macromolecular Chemistry (IOMC), Friedrich Schiller University Jena, Humboldtstrasse 10, 07743 Jena, Germany. ${ }^{4}$ Molecular Hemostaseology, Jena University Hospital, Bachstraße 18, 07743 Jena, Germany. ${ }^{5}$ Department of Biological Sciences and Center for Biomedical Engineering and Science, University of North Carolina at Charlotte, Charlotte, North Carolina 28223, USA. ${ }^{6}$ Institute of Biochemistry I, Jena University Hospital, Friedrich Schiller University, 07743 Jena, Germany. ${ }^{7}$ Chair for Biological and Medical Imaging, Technical University Munich and Helmholtz Zentrum München, Ingolstädter Landstrasse 1, 85764 Neuherberg, Germany. ${ }^{8}$ Leibniz Institute for Photonic Technology Jena, Albert-Einstein-Strasse 9, 07745 Jena, Germany. ${ }^{9}$ Abbe Center of Photonics, Friedrich Schiller University, Max-Wien-Platz 1, 07743 Jena, Germany. ${ }^{\star}$ These authors contributed equally to this work. $\dagger$ These authors share the senior authorship. Correspondence and requests for materials should be addressed to M.B. (email: michael.bauer@med.uni-jena.de).
} 
$\mathrm{D}$ rugs based on RNA interference (RNAi) reflect a promising class of therapeutics to interfere with virtually any protein-coding messenger RNA (mRNA), thus opening up new treatment strategies for targets that are, at present, deemed not amenable to drug development ${ }^{1-4}$. In contrast to small molecules with a wide range of different physicochemical properties, the chemical similarity of short interfering RNAs (siRNAs) permits the development of platform technologies ${ }^{5-7}$.

The most prominent obstacle in translating RNAi-based approaches into a new class of therapeutics is a specific delivery and release of siRNAs to the targeted cells, tissues and organs, particularly important when off-target effects need to be avoided $^{8,9}$.

siRNAs are negatively charged, hydrophilic molecules that must overcome the hydrophobic plasma membrane ${ }^{10,11}$ to reach-in a multistep process-the RNA-induced silencing complex as their presumed site of action ${ }^{12}$. Cationic lipids are described as potential carriers to overcome electrostatic repulsion by binding and neutralising the negative charge of siRNAs simultaneously ${ }^{13}$. In addition, polycationic derivatives can effectively condense nucleic acid cargo for transfer into the cells, especially polyethylenimine (PEI), which can be considered a golden standard polymer owing to its high buffering ability for endosomal escape of siRNA to be delivered ${ }^{14}$. Penetrating the cell membrane may be achieved by conjugating siRNAs to small chemical moieties, such as sugar moieties, peptides or lipids ${ }^{11,15,16}$. These conjugation approaches enhanced the cellular entry of siRNAs, but confer organ selectivity only to a very limited extent ${ }^{16}$.

To fully exploit the potential of these strategies innovative delivery systems allowing active targeting are required, particularly if the systemic delivery of siRNA to internal organs is desired. Approaches previously applied to deliver siRNAs include viruses and non-viral vectors with inherent differing efficiency and toxicity ${ }^{17,18}$; however, these are far from satisfactory ${ }^{19}$. Depending on the application, target tissue and disease, versatile delivery strategies are of the utmost importance. Ultimately, a theranostic approach ${ }^{20,21}$ is desirable, whereby an upstream diagnostic test could account for any inter-individual variability in carrier and payload uptake such that the type and/or dose of the carrier can be individualised.

Owing to its broad metabolic repertoire, the liver, more specifically hepatocytes, constitute particularly important targets for siRNA delivery ${ }^{22,23}$. A well-characterised way of delivering siRNA cargos utilises liposomes that directly release the siRNA into the cytoplasm after fusing with the plasma or endosomal membrane ${ }^{24}$. This allows, albeit with limited cellular selectivity, delivery of siRNA into the liver ${ }^{25,26}$.

A higher selectivity could potentially be achieved by other uptake mechanisms, such as receptor-mediated endocytosis of polymer-based nanoparticles (NPs). Uptake transporters with organ-specific expression pattern are present in epithelial cells, for example, organic anion transporting polypeptides (OATP) ${ }^{27,28}$ found within the basolateral membrane of hepatocytes. Polymethine dyes, such as indocyanine green (ICG), which are ligands for these transporters, have been used for decades to assess hepatic excretory function ${ }^{29-32}$.

Here we report that polymethine dyes, which differ regarding their physicochemical characteristics, are eliminated with high selectivity via the hepato-biliary or renal route. These dyes can be covalently bound to polymers conferring selectivity for organspecific uptake transporters to subsequently formed siRNAloaded NPs. As a result, the dye-NP conjugate (DY-[NP]) reflects an escort system, for which imaging strategies to monitor uptake and clearance can be developed. This could allow the design of a platform-technology for theranostic delivery of RNAi therapeutics to the liver and, potentially, the kidney.

\section{Results}

NIR fluorescent dyes for functionalisation of nanoparticles. We initially screened different polymethine dyes based on benzopyrylium or indolium salts with solubilising groups, that is, sulfonic residues. Although dyes containing four sulfonic residues displayed preferential renal elimination, dyes containing only one sulfonic residue were subject to hepatobiliary excretion (Fig. 1a and Supplementary Table 1). Among the studied compounds, DY-780 and DY-635 were excreted preferentially via the bile. While the optical properties of DY-780 proved to be suitable for imaging by multispectral optoacoustic tomography (MSOT), DY635 revealed superior properties to monitor hepatobiliary clearance by intravital microscopy (IVM) (Fig. 1b). Several more hydrophilic dyes, such as DY-704 showed promising pharmacokinetic properties to target renal tubular epithelia (Fig. 1b).

To develop a theranostic delivery system for liver parenchyma, we selected DY-635 and characterised its affinity to basolateral transporters in a heterologous expression system. While DY-635 exhibited moderate affinity to several important transporters, such as the sodium-taurocholate cotransporting polypeptide (NTCP), its affinity to OATP1B1 and OATP1B3 in transfected HEK cells substantially exceeded that of the Food and Drug Administration (FDA) proposed ligand for drug development, rifampicin (ref. 33, Fig. 1c). Finally, DY-635 uptake by freshly isolated primary mouse hepatocytes was found to be rapid, temperature sensitive and inhibited dose-dependently by cyclosporine $\mathrm{A}$ with an $\mathrm{IC}_{50}$ of $379 \mathrm{nM}$ (Fig. 1d).

Polymer-synthesis and formulation of nanoparticles. DY-635 was covalently coupled to acid terminated poly-lactic-co-glycolic acid (PLGA) via 1-ethyl-3-(3-dimethylaminopropyl)carbodiimide hydrochloride (EDC) chemistry. Covalent coupling of copolymer and dye was confirmed by size exclusion chromatography using $\mathrm{UV} /$ visible and refractive index (RI) detectors (Supplementary Fig. 1a) as well as UV/visible spectroscopy (Supplementary Fig. 1b). The calculated labeling efficiency for conjugation was $77 \%$, meaning that every 130th (aimed: approximately every 100th) polymer chain bears a label. The subsequent NP formulation was accomplished by nanoprecipitation from pure PLGA (NP), PLGA with coupled dye (DY-635[NP], DY$704[\mathrm{NP}])$ and PLGA with coupled dye and loaded with additional dyes (DY-635[NP](DY-780), DY-635[NP](NileRed), DY$635[\mathrm{NP}](\mathrm{ICG})$ ). For in vivo gene silencing, siRNA was complexed with linear PEI of low molar mass $(10 \mathrm{kDa}$; obtained by hydrolysis of poly(2-ethyl-2-oxazoline)). These complexes as well as ICG dye were encapsulated by emulsion techniques into a polymeric shell of the respective polymer (Fig. 2a). Particle surface fluorescence quenching was performed using trypan blue to demonstrate expression of the near infrared (NIR) dye on the particle surface, as this represents an essential feature for specific uptake. The investigations showed a decrease of fluorescence by nearly $75 \%$ (Fig. 2b). This indicates strongly that the dye is exposed to the particle surface and supports the notion that the dye moiety is responsible for targeting properties. The formed NPs were further characterised in detail by different methods to investigate their size, shape and surface charge (Table 1). The siRNA was encapsulated achieving an efficiency of $87.4 \pm 2.5 \%$ (Table 1) as determined by photometric phosphate quantification. Scanning electron microscopy (SEM) measurements (Fig. 2c-e) showed no significant differences between [NP], DY$635[\mathrm{NP}](-)$ and DY-635[NP](siRNA). The zeta potentials, representing the surface charges of the NPs, are shown in Fig. 2c, 


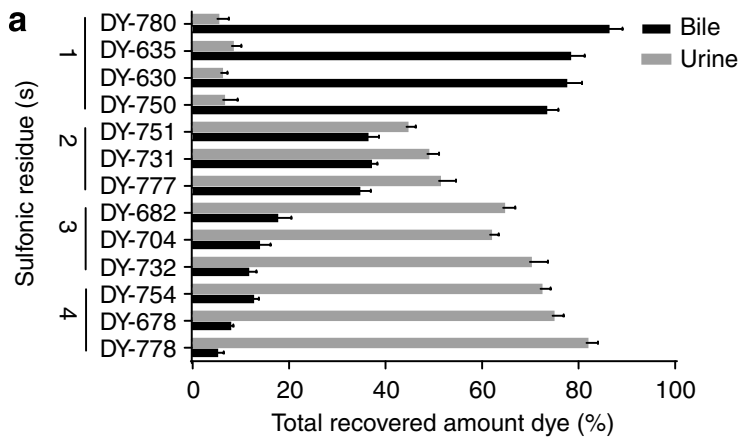

b

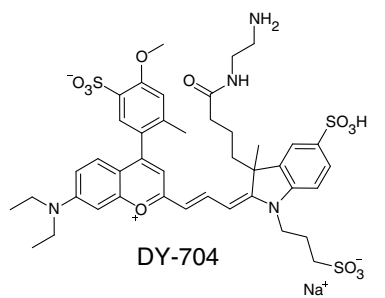
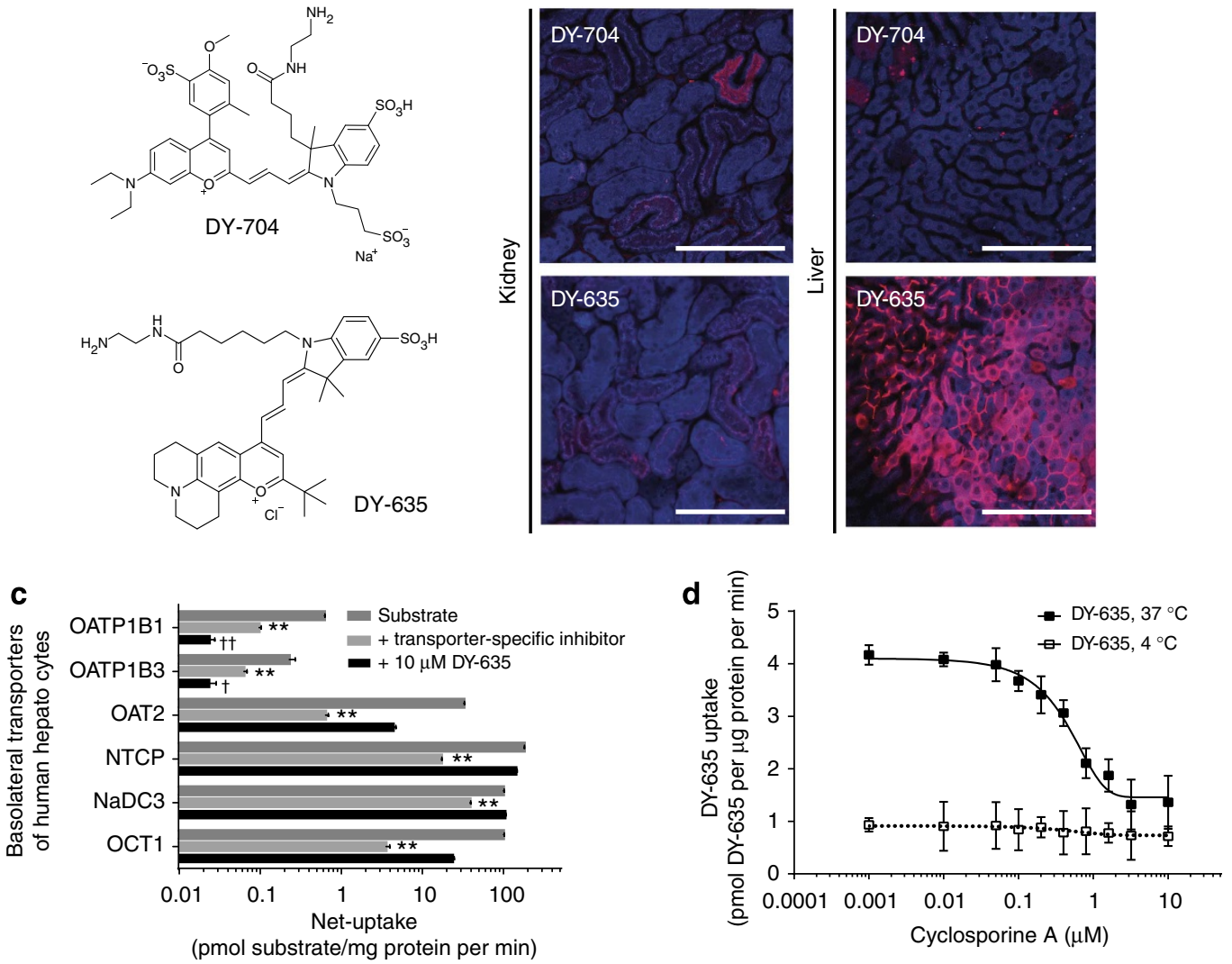

Figure 1 | Selection of polymethine dyes with organ-selective elimination. (a) A variety of polymethine dyes with varying numbers of sulfonic residues were screened in rats regarding net organ-specific clearance by liver and kidneys and their applicability for biophotonic detection, for example, by IVM; $(n=3$; mean \pm s.e.m.). (b) Structures of DY-635, a dye with preferential hepatobiliary clearance and DY-704 with preferential renal elimination and their cell-specific uptake as visualised by intravital laser scanning confocal microscopy in liver and kidney (scale bars $150 \mu \mathrm{m}$ ). (c) DY-635 was further characterised regarding its affinity to transporters responsible for uptake of substrates into the liver in a heterologous expression system (HEK cells). While DY-635 exhibited moderate affinity to several transporters, its affinity to OATP1B1 and OATP1B3 exceeded that of rifampicin or cyclosporine A, that is, the FDA-proposed competitors for drug development purposes. Bars show mean \pm s.e.m.; significance was tested by one-way ANOVA followed by Tukey's test; ${ }^{* *}$ indicates $P<0.01$ for uptake of the substrate in the presence of DY-635 (hatched bars) or the FDA-proposed competitor (black bars) compared with substrate alone. $\dagger$ and $\dagger \dagger$ indicate $P<0.05$ and $P<0.01$, respectively, for the comparison between DY- 635 and the FDA-proposed competitor. (d) Uptake of DY-635 by primary murine hepatocytes is temperature sensitive and inhibited by cyclosporine $\mathrm{A}$ in a dose dependent manner with an IC ${ }_{50}$ of $379 \mathrm{nM}$.

indicating negative charge for pure PLGA, DY-635[NP]s and loaded DY-635[NP]s (Table 1).

DY-635[NP](siRNA) were investigated in further detail using asymmetric flow field-flow fractionation (AF4). A monomodal and nearly monodisperse distribution with low particle polydispersity index (1.09) (Supplementary Fig. 1c), a radius of gyration $\left(R_{\mathrm{g}}\right)$ of $70 \mathrm{~nm}$ and a hydrodynamic radius $\left(R_{\mathrm{H}}\right)$ of $90 \mathrm{~nm}$, which is in accordance to batch dynamic light scattering (DLS) measurements, could be determined. The shape ratio $\left(R_{\mathrm{g}} / R_{\mathrm{H}}\right)$ of 0.78 obtained by AF4 measurements (Supplementary Fig. 1d) corresponds to the theoretical value of a hard sphere $(0.775)^{34}$. To confirm that the siRNA-PEI complexes are encapsulated inside the NPs and not aggregated onto their surface, individualised transmission electron microscopy (TEM) (Fig. 2f) as well as cryo-TEM measurements (inset Fig. 2f) were performed. The PEI core of DY-635[NP] (siRNA) was counterstained with $\mathrm{Cu}^{2}+$ ions, demonstrating that the siRNA-PEI complexes were located inside the NPs. Furthermore, bio- and haemo-compatibility were studied showing neither haemolysis nor aggregation of erythrocytes (data not shown), supporting the successful encapsulation of the siRNA-PEI 
a
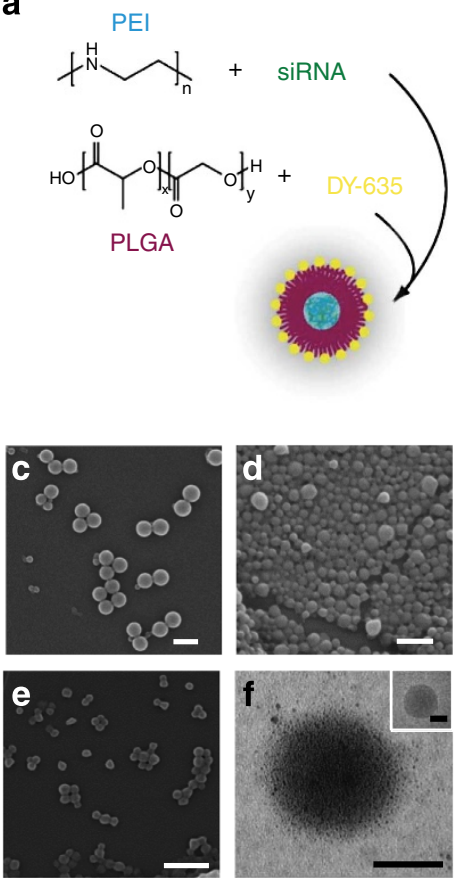

b

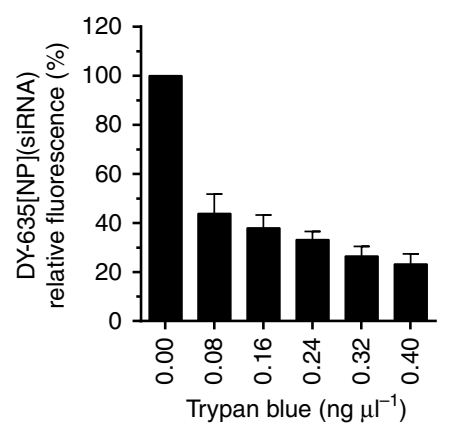

9

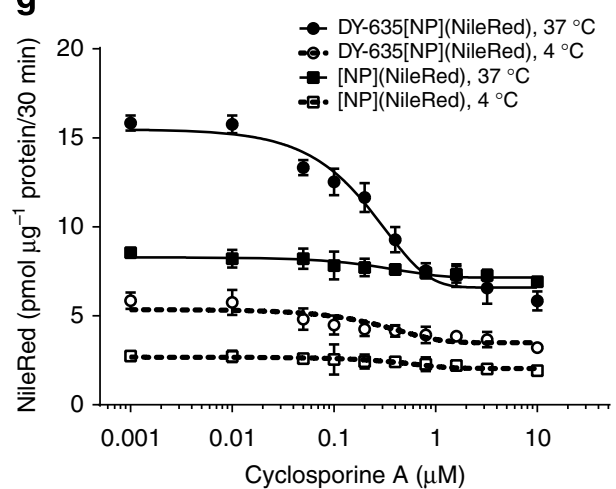

Figure 2 | Formation and characterisation of NPs. (a) Dye-functionalisation of NPs. PLGA is labelled with DY-635 via EDC coupling, siRNA is complexed with LMW PEI, and NPs are formed by double emulsion procedure. (b) Surface fluorescence quenching of DY-635[NP]( - ) indicates that DY-635 is exposed at the NP surface $(n=3$, mean \pm s.e.m.). (c-e) Electron microscopy of NPs ([NP]( - ), DY-635[NP]( - ), DY-635[NP](siRNA) reveals regular round shape (scale bars $500 \mathrm{~nm}$ ). (f) Higher power TEM of DY-635[NP](siRNA) after treatment with $\mathrm{Cu}^{2+}$-ions (inset shows the corresponding cryoTEM image) suggests siRNA is encapsulated and not aggregated to the surface (scale bars $100 \mathrm{~nm}$ ). (g) Uptake of DY-635 functionalised NPs is enhanced compared with non-functionalised NP and, unlike that of non-functionalised NPs is inhibited in a dose-dependent manner by cyclosporine $\mathrm{A}_{\text {with }}$ an IC $\mathrm{C}_{50}$ of $209 \mathrm{nM}$, similar to the uptake of free DY-635.

Table 1 | Characterisation of nanoparticles.

\begin{tabular}{lcccc} 
& Targeting moiety & Active payload & Size $^{\star}$ (d in nm) & Surface charge $^{\dagger}$ (in mV) \\
\hline$[N P](-)$ & - & - & $210 \pm 50$ & $-7 \pm 2$ \\
FNP](siRNA) & - & siRNA & $190 \pm 32$ & $+78 \pm 5$ \\
{$[N P](N i l e R e d)$} & - & NileRed & $196 \pm 24$ & $-25 \pm 18$ \\
{$[N P](I C G)$} & - & ICG & $145 \pm 45$ & $-24 \pm 6$ \\
DY-635[NP](-) & + & - & $170 \pm 5$ & $-13 \pm 5$ \\
DY-635[NP](siRNA) & + & siRNA & $176 \pm 22$ & $-11 \pm 6$ \\
DY-635[NP](NileRed) & + & NileRed & $142 \pm 18$ & $-19 \pm 7$ \\
DY-635[NP](ICG) & + & ICG & $151 \pm 32$ & $-42 \pm 6$ \\
DY-704[NP](-) & + & - & $155 \pm 37$ & $0.78^{\S}, 87.4 \pm 2.5 \% \|$ \\
\hline
\end{tabular}

*Determined by DLS.

$\dagger$ Zeta potential.

†Complexed with LMW PEI, N/P10.

\$Rg/Rh determined by AF4.

||Encapsulation efficiency.

complexes. We then tested whether DY-635 conjugated to NPs would retain selective uptake by primary hepatocytes. Similar to what was observed with the free dye (Fig. 1d) DY-635[NP]( - ) were rapidly accumulated in a temperature sensitive manner by primary hepatocytes and dose-dependently inhibited by cyclosporine $\mathrm{A}$ with an $\mathrm{IC}_{50}$ similar to that observed for inhibition of uptake of the free dye (209 nM, Fig. $2 \mathrm{~g}$ ).

Body-distribution and traceability of DY-635[NP]. Having demonstrated that organ specificity can be transferred to dye-functionalised NPs, body distribution of DY-635[NP], carrying contrast agents as cargo, was analysed regarding active versus passive targeting properties. Athymic Nude-Foxn $1^{\text {nu }}$ mice bearing a highly vascularised MDA-MB-231 human breast cancer xenograft were injected with DY-635[PLGA](DY-780) and analysed after cryoslicing by low-power fluorescence microscopy. Although liver, gall bladder and subsequently the intestinal lumen showed dye signal suggesting uptake by hepatocytes followed by biliary excretion, no dye signal was detected in the tumour (Fig. 3a). This is indicative of primarily active targeting to liver parenchyma rather than passive targeting even in the presence of 

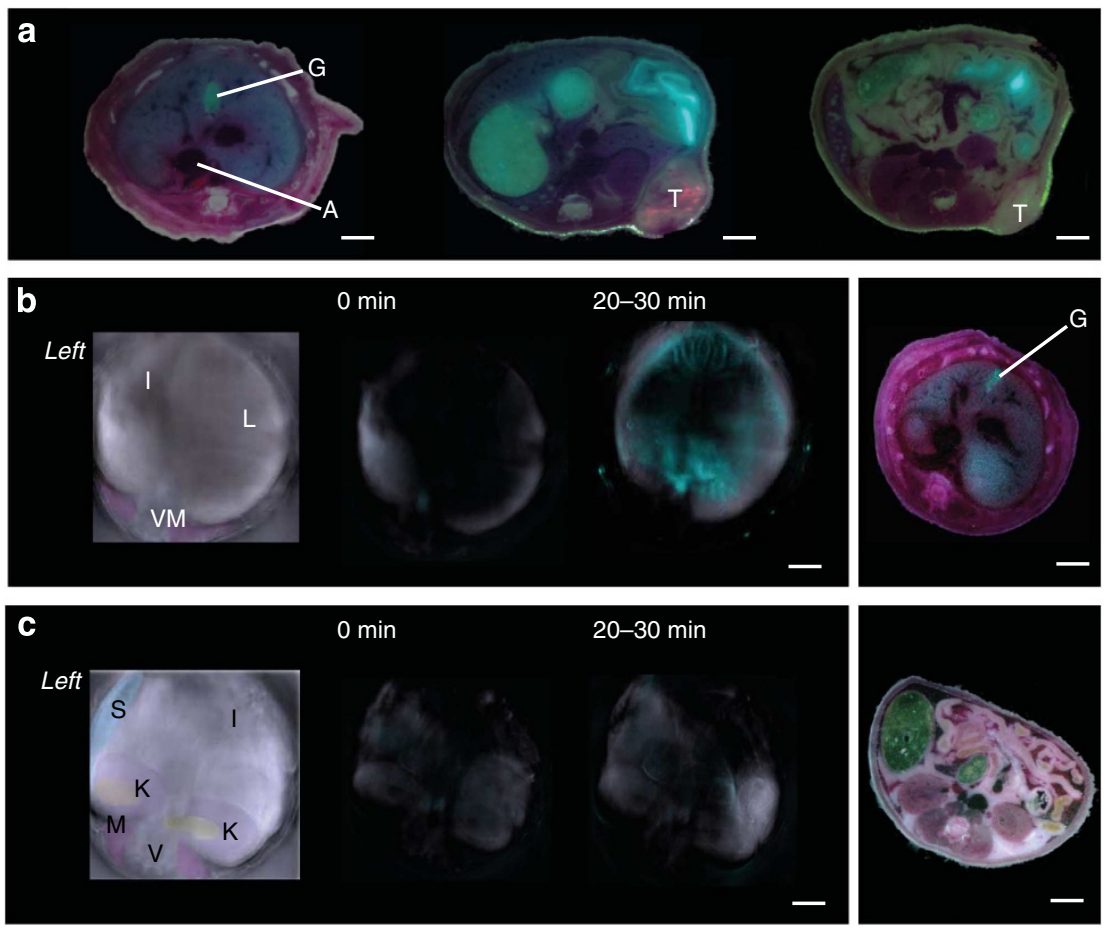

Figure 3 | Biodistribution of NPs. (a) Body distribution of dye-functionalised NPs with an infrared cargo (DY-635[NP](DY-780)) in an athymic NudeFoxn $1{ }^{\text {nu }}$ mouse bearing a MDA-MB-231 human breast cancer xenograft (' $\mathrm{T}^{\prime}$ ) confirmed selective uptake and excretion via the hepatobiliary route as reflected in accumulation of the dye in the gall bladder (' $G$ '). $(\mathbf{b}, \mathbf{c})$ CD1 mice injected with the same NP and assessed by multispectral optoacoustic tomography acquiring image data from several wavelengths $(690,710,750,770,780,800,810$ and $850 \mathrm{~nm}, 4$ frames/wavelength, $0.1 \mathrm{~mm}$ step size) over an abdominal area of $1.7 \mathrm{~cm}$ using a frequency of $54.55 \mathrm{kHz}$ (scale bars $5 \mathrm{~mm}$ ). (b) Sections through the upper abdomen are single-spectral that elucidate anatomical structures (left panel; 'I' intestines, ' $L$ ' liver, ' $V$ ' vertebral column, ' $M$ ' autochthonous back muscles and ' $A$ ' aorta) or processed multispectrally to visualise the dye cargo (DY-780) immediately prior to injection ( $0 \mathrm{~min}$ ) or during early uptake (20-30 min after injection). Far right panel: Corresponding cryosection of the upper abdomen; ' $G$ ' indicates gall bladder containing the dye. (c) Subsequent sections through the lower abdomen processed for structural information (left panel; in addition the kidneys (' $\mathrm{K}$ ') and spleen (' $\mathrm{S}$ ') are visible) middle and right panels are processed to visualise the dye cargo. The IR dye is restricted to the upper abdomen and depicts liver and biliary tree.

a highly vascularised tumour. Subsequently we tested whether the optical properties of DY-780 would allow non-invasive imaging of organ distribution using MSOT in anaesthetised, healthy CD1 mice. Consistent with the uptake pattern in nude mice, a DY-780derived signal in liver parenchyma, biliary tree and gut could be detected by MSOT indicating that hepatocytes take up and secrete DY-780 into bile (Fig. 3b,c). Further experiments were conducted to confirm the need for a hepatoselective dye to target liver parenchyma. MSOT confirmed unspecific uptake of all applied NPs in spleen and to a much lesser extent in the liver (Fig. 4a). While NPs with DY-635 as targeting moiety were extensively accumulated by the liver, either absence of dye or functionalising with DY-704, that is, a dye that is not a substrate for hepatocyte transporters, failed to show active targeting to the liver. Moreover, in vivo laser scanning microscopy indicated that bare NPs carrying nile red were taken up by the liver and found almost exclusively in sinusoidal lining cells (primarily Kupffer cells). In contrast, DY-635 functionalised NPs were taken up by hepatocytes with the nile red cargo retained in the cytoplasm and the DY-635 transported into the bile (Fig. 5a,b). Thus, the NPs maintain their affinity to hepatic uptake transporters and allow tracking of drug delivery by biophotonic strategies.

Uptake mechanisms of DY-635 and DY-635[NP] (-). A microfluidic-assisted organoid that mimics the sinusoidal anatomy enabling us to study uptake kinetics of a human hepatocellular cell line (HepaRG) in the presence of human umbilical vascular endothelial cells (HUVEC) under static or flow conditions was used to assess mechanisms of uptake (Supplementary Fig. 2). As shown in Fig. 6a,b, uptake of DY-635[NP] ( - ) was significantly increased under conditions of flow compared with the static culture. In the presence of flow, the pressure gradient in the flow chamber results in efflux of perfusate (including NPs) out of the 'vascular' space into the space surrounding the HepaRG cells (analogue of space of Disse in vivo) at the inflow and back into the vascular space at the outflow. In this way, DY-635[NP] are delivered to the HepaRG cells similar to what occurs in the liver in vivo. In a co-culture of HepaRG and HUVEC cells we could also demonstrate selectivity of DY-635[NP] $(-)$ to hepatocytes and inhibition of NP uptake by cyclosporine A and Pitstop-2 supporting an OATP-dependent clathrin-mediated endocytosis (Fig. 6b,c). Basal uptake of NP made from unfunctionalised PLGA and loaded with NileRed for detection was not affected by cyclosporine A (Fig. 6d).

Since high selectivity for hepatocytes was confirmed for DY$635[\mathrm{NP}](-)$ and because liver parenchyma could secrete free DY-635 into bile ${ }^{32}$, we performed in vivo pharmacokinetic measurements of DY-635[NP] $(-)$ to assess (1) plasma disappearance, (2) hepatocellular clearance and (3) appearance of the dye in collected bile. After central venous administration of DY-635[NP] $(-)$, plasma kinetics in arterial blood revealed a peak at $4 \mathrm{~min}$ after administration. Disappearance followed an exponential decay with biliary excretion reaching its maximum at $15 \mathrm{~min}$ (Fig. 7a). Monitoring of parallel urine samples was consistent with almost exclusive secretion of DY-635 from 


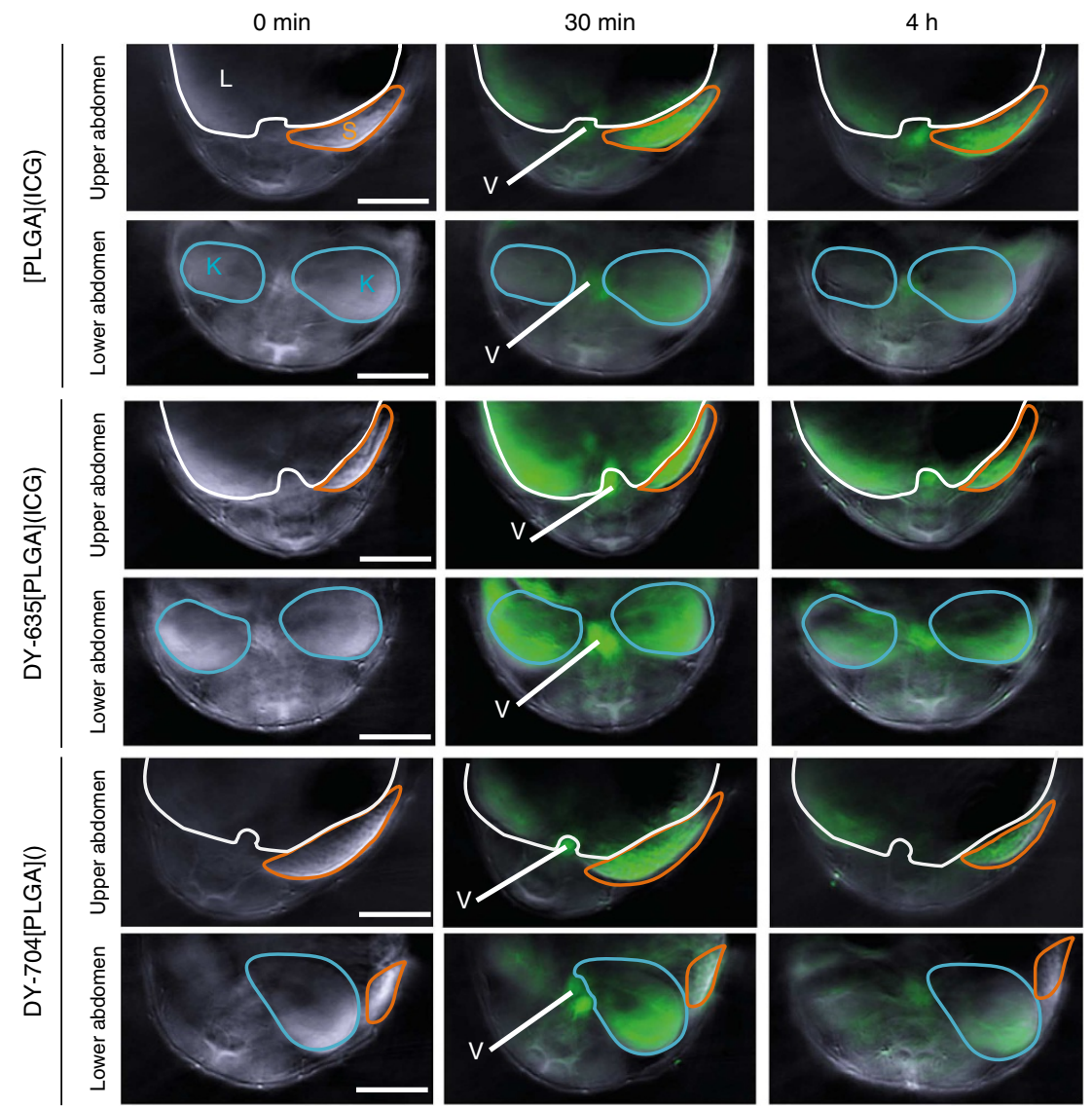

Figure 4 | Organ distribution of dye-functionalised NP. MSOT images of non-functionalised NP or NP functionalised with DY-635 with ICG as cargo or functionalised with DY-704. The dye-moiety (in case of DY-704) or ICG cargo (in the case of DY-635 or non-functionalised NP) were used for imaging by MSOT; NP are shown in green irrespective of the dye moiety. All NP were identified in the circulation ( $V=$ retroperitoneal vessels) and showed uptake by spleen (S) indicating phagocytic clearance; however, only DY-635 functionalised NP showed significant uptake by the liver (L), whereas DY-704 functionalised NP had an increased accumulation in the kidneys (K) (scale bars $5 \mathrm{~mm}$ )
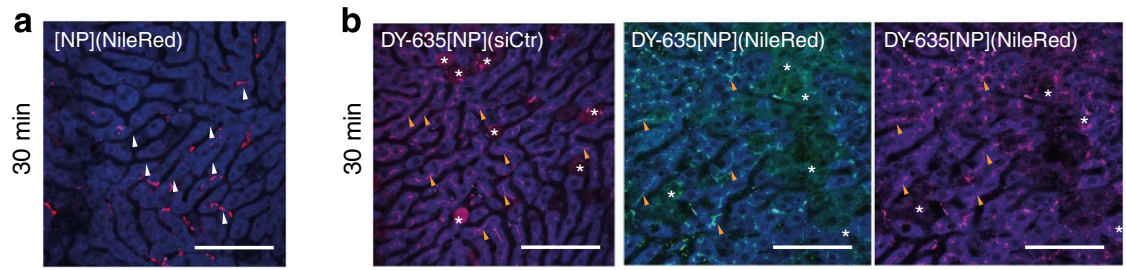

Figure 5 | Cell-type distribution of dye-functionalised NP. (a) In vivo laser scanning confocal images of mouse liver (blue) 30 min following injection of bare PLGA-NPs containing nile red (red). These appear almost exclusively in sinusoidal lining cells (Kupffer cells, white arrow; scale bar $100 \mu \mathrm{m}$ ),

(b) NP functionalised with DY-635, in contrast, accumulate in hepatocytes where the NileRed cargo (green) and DY-635 (red) are found either in bile canaliculi (orange arrows) or in hepatocyte cytoplasm $\left(^{\star}\right)($ scale bars $100 \mu \mathrm{m})$.

DY-635[NP]( - ) via the hepatobiliary route. When biliary recovery of DY-635 was quantified, the ratio of secreted DY-635 to injected DY-635 bound to DY-635[NP] $(-)$ exceeded $90 \%$ after 45 min (Fig. $7 \mathrm{~b}$ ). A change in the relative fluorescence intensity decrease in the hepatocyte from an exponential decay (free dye) to an almost linear kinetic after injection of DY-635[NP] $(-)$ was observed supporting the assumption that DY-635 and DY-635[NP] are processed differently and that rate limiting intracellular reactions have to occur prior to secretion of the dye (Fig. 7c). Sublobular distribution of DY-635[NP] $(-)$ with liver tissue, as shown in Fig. $7 d$, confirmed the highest concentrations around central veins with a steady decrease towards the lobular periphery early upon injection (Fig. $7 \mathrm{~d}$, upper and middle panel).
The fluorescence signal subsequently displayed a spreading towards the periportal region (Fig. $7 \mathrm{~d}$, lower panel).

To assess the potential use of the free dye to predict uptake of DY-635[NP] in a theranostic system, we injected DY-635 and DY-635[NP] ( - ) sequentially. After DY-635 injection an increase in fluorescence intensity was observed followed by an exponential decay. Administration of DY-635[NP] ( - ) 20 min after DY-635 injection resulted in a second peak with a linear decay as shown for the individual compounds (Fig. 7e), whereas distribution of DY-635 and DY-635[NP] were similar (Fig. 7f)

Cell type-specific RNAi of Hmgcr using DY-635[NP] (siHMGCR). Next, we performed functional analysis of 

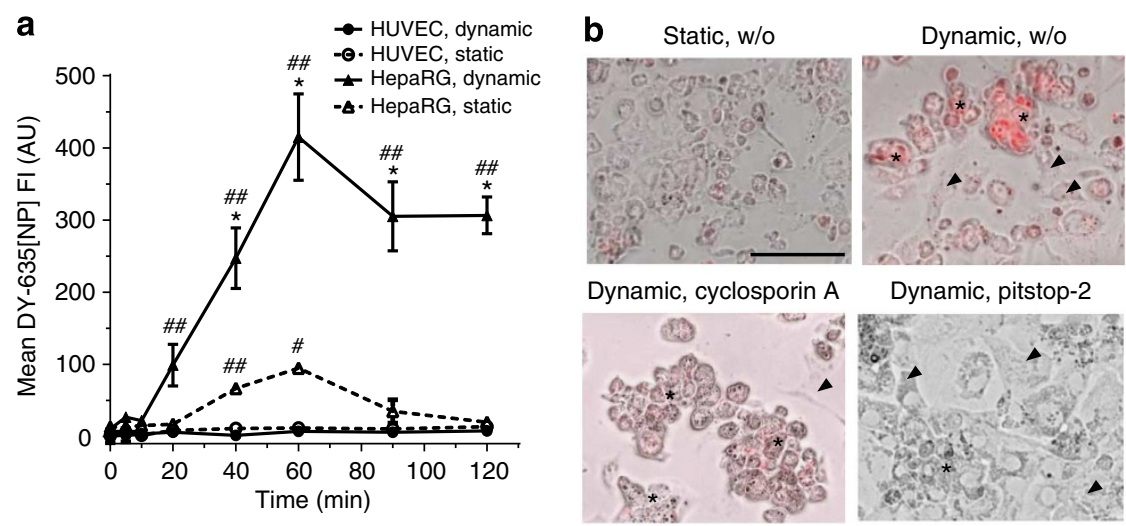

Dynamic, pitstop-2
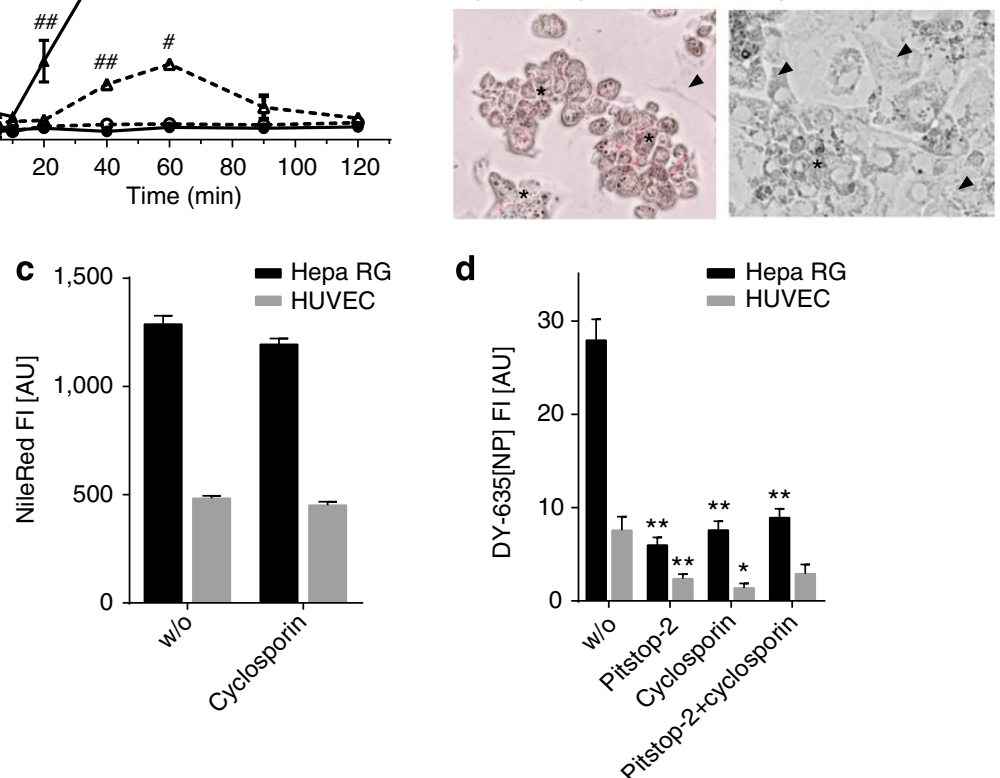

Figure 6 | Uptake of NPs exposing DY-635 on their surface in vitro. (a) A microfluidic-assisted 'organoid' composed of co-cultured HepaRG and HUVEC demonstrates an increased uptake by the hepatocytes preferentially if cells are subjected to flow conditions ('dynamic'). Under flow conditions, this organoid better recapitulates the function of the liver in vivo by circulating fluid containing NP through the equivalent of the space of Disse.

(b) Representative overlay of brightfield and fluorescence images of HepaRG cells differentiated to hepatocytes (stars) or endothelia like (arrow head) cells cultured under static or dynamic conditions reflect increased uptake of DY-635[NP]( - ) (red) after 90 min of incubation if cells are subjected to flow. This uptake can be inhibited by cyclosporine A as well as Pitstop-2. DY-635[NP]( - ) uptake was assessed by epi-fluorescence microscopy (Cy5 channel); (scale bar $100 \mu \mathrm{m}$ ). To characterise cell selectivity and uptake mechanisms, further experiments were conducted. (c) Uptake of DY-635[NP]( - ) is inhibitable by cyclosporine A, a ligand for OATPs and NTCP in HepaRG but not in HUVEC that do not express these transporters; inhibition by Pitstop-2 suggests clathrin-mediated endocytosis as molecular mechanism of cellular uptake. (d) Cyclosporine A failed to affect the overall less pronounced basal uptake of $[N P]$ (nile red) that are not exposing DY-635 on their surface. For the statistical analysis a generalised mixed model was applied, taking into account dependent (time, flow condition) and independent (cell type) data. Post hoc analysis was performed using Tukey's test. In panel (a) * indicates $P<0.05$ comparing respective static versus dynamic conditions; \# indicates $P<0.05$, \#\# indicates $P<0.01$ for the comparison between HepaRG and HUVEC. In panel (d), ${ }^{*}$ and ${ }^{* \star}$ indicate $P<0.01$ and $P<0.01$ respectively, for the uptake of DY-635[NP]( - ) in HepaRG or HUVEC in the absence of a co inhibitor (w/o) compared with the uptake in presence of cyclosporine A or Pitstop-2.

DY-635[NP](siRNA) delivery of siRNA in Hepa1-6 cells. In comparison to untreated controls, we observed a decrease in hmgcr mRNA-levels of $75 \%$ after $16 \mathrm{~h}$ and of $60 \%$ after $24 \mathrm{~h}$. In contrast, DY-635[NP](siCtrl) loaded with a control siRNA showed some stimulatory effect on HMGCR mRNA at 16 and $24 \mathrm{~h}$ after treatment so that the total (net) inhibitory effect of hmgcr RNAi was even more substantial in comparison to DY635[NP] (siCtrl) at both 16 and $24 \mathrm{~h}$ (Fig. 8a).

As successful RNAi was critical to our therapeutic strategy, we next validated the RNAi effects directly at the protein level. We first demonstrated the knockdown of the catalytic domain of HMGCR fused to green fluorescent protein (GFP) and expressed under a cytomegalovirus-promotor from vectors that additionally drive either specific RNAi or scrambled RNAi in HEK293 cells using quantitative immunoblotting (Fig. 8b).

Second, we directly assessed the knockdown of endogenous HMGCR by quantitative immunofluorescence analyses in HepG2 cells. Both RNAi constructs led to a significant reduction of antiHMGCR immunosignals (Fig. 8c; Supplementary Fig. 3). We then tested whether knockdown with DY-635[NP](siHMGCR) would be effective in vivo using a mouse model. After two injections via a venous port, liver tissue was analysed. An absolute decrease of $h m g c r$ mRNA of $70 \%$ versus untreated animals was achieved (Fig. 8d). This decrease in mRNA was functionally significant, as DY-635[NP](siHMGCR) treatment-mediated decrease of $h m g c r$ mRNA resulted in a significant reduction of plasma cholesterol compared with the controls (Fig. 8e). Again it was observed that treatment with DY-635[NP](siCtrl) lead to an up-regulation of $h m g c r$ accompanied by an increase in $h m g c r$ mRNA (7d) and plasma-cholesterol levels (Fig. 8e). This is likely the result of low level activation of lipid metabolic pathways known to be associated with transient penetrations of the cell wall $^{35}$.

\section{Discussion}

In this study, we characterised multifunctional NPs generated by covalent conjugation of a fluorescent dye with known hepatobiliary clearance to a biodegradable polymer (PLGA) as a carrier system for PEI-complexed RNAi therapeutics ${ }^{36-39}$. The highly 

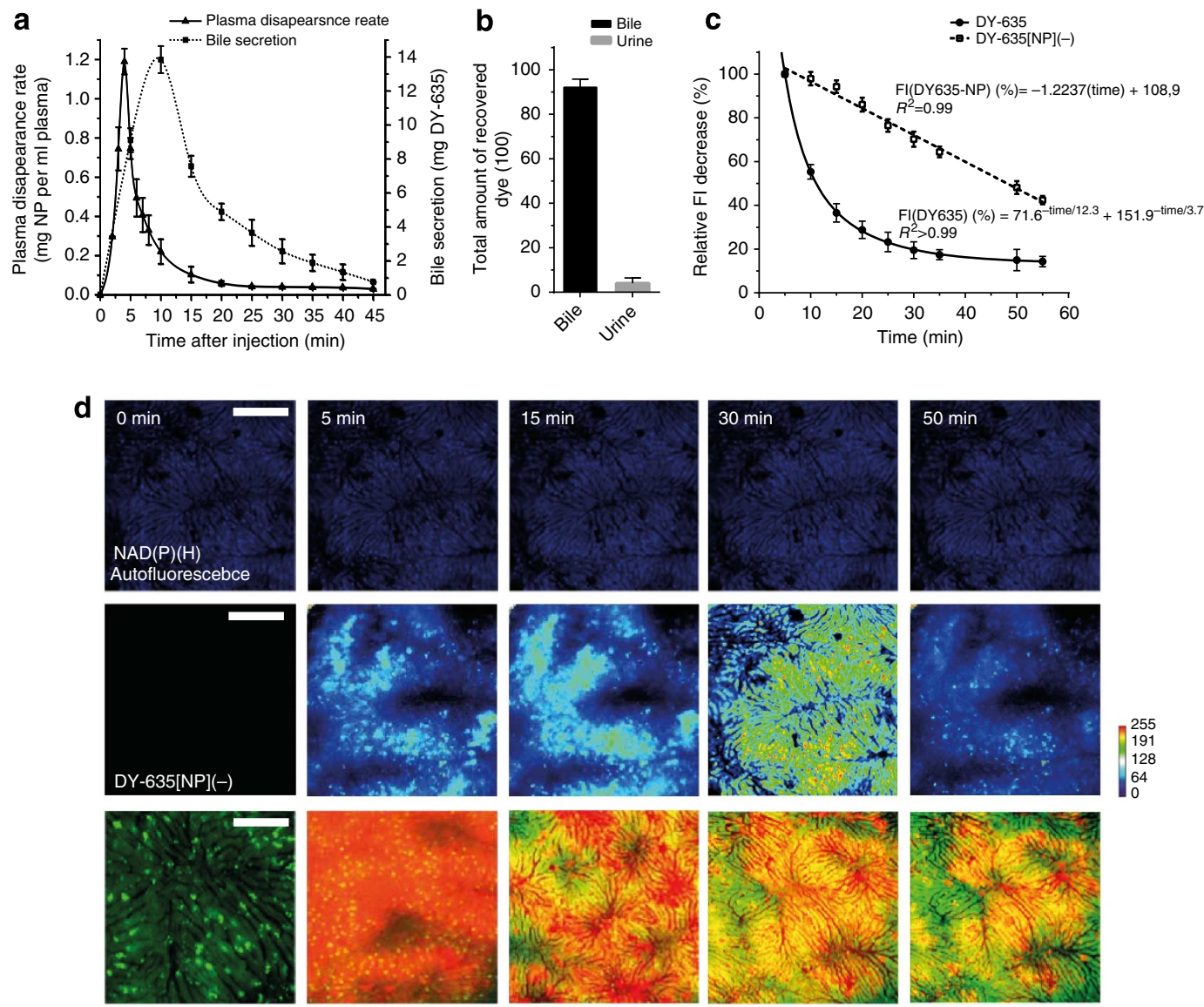

e

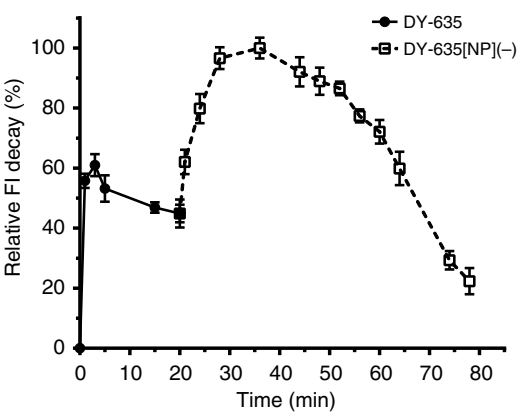

f DY-635, 8 min $\quad$ DY-635[NP](-), 8 min

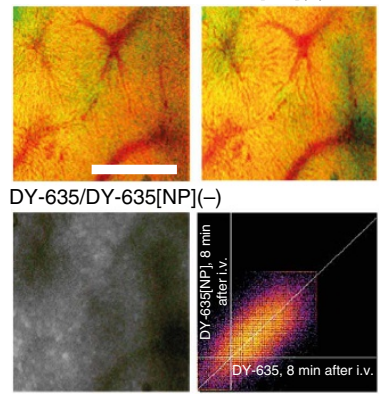

Figure 7 | Pharmacokinetics of DY-635[NP]( - ). (a) Time course of arterial plasma concentration after central venous application of DY-635[NP]( - ) (left ordinate) at time ' $O$ ' and appearance of the desorbed dye in bile (right ordinate) ( $n=3$, mean \pm s.e.m). (b) Percentage of recovered free dye in bile and urine after administration of DY-635[NP]( - ). (c) Fluorescence decay over liver parenchyma in the Cy 5 channel after administration of unbound DY-635 or DY-635[NP] $(-)$ revealing exponential decay for the free dye and almost linear decay for DY-635[NP]( - )-associated fluorescence $(n=3$ per 25 ROIs per $\mathrm{n}$; mean \pm s.e.m.). (d) Intravital epifluorescence microscopy to visualise acinar distribution of DY-635[NP]( - ). Upper panel background fluorescence of the liver (blue). Middle panel: Heatmap (blue: low intensity, red: high intensity) reflecting signal distribution which is associated with the pericentral region of the liver lobule early upon injection of DY-635[NP]( - ) and spreads towards midzonal and even periportal region over time. Lower panel: corresponding false colour images of association of DY-635-associated fluorescence (red) with liver parenchyma (green): fluorescence is restricted to the vascular compartment early upon injection ( $5 \mathrm{~min}$ ); yellow signal indicating colocalisation of dye with liver parenchyma is increasingly observed over time. (scale bars $100 \mu \mathrm{m})$. (e) Theranostic use of the free dye DY-635 to predict subsequent uptake of DY-635[NP]( - ). DY-635 was injected at time '0' followed by injection of DY-635[NP]( - ) at $20 \mathrm{~min}$. (f) Intensity of fluorescence signal over liver parenchyma; right panel: signals obtained upon injection of free dye and dye-functionalised nanoparticles are correlated. ( $n=2,20$ ROls per $n$; mean \pm s.e.m.) (scale bar $100 \mu \mathrm{m})$.

organ-selective uptake of the NIR dye was maintained after covalent binding to PLGA-NPs. Furthermore, the NIR dye moiety allowed monitoring of distribution, uptake and clearance using biophotonic approaches. Assessing the pharmacokinetics of DY$635[\mathrm{NP}](-)$ confirmed hepatobiliary clearance exceeding $90 \%$ in $<1 \mathrm{~h}$. The conjugate thus represents a fundamentally new class of theranostic delivery systems with a highly efficient active-targeted delivery and the option, based on the preceding injection of the free dye, to predict the tissues that will eventually take up a nanoformulated drug before it is administered. Since these dyes can be modified with regards to their physicochemical properties (as shown exemplarily for DY-704 which is eliminated primarily via the kidney), a platform technology to deliver dyefunctionalised NPs to a variety of cells and tissues expressing 
a

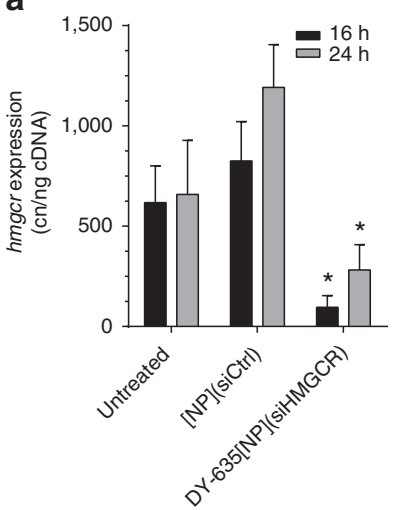

b

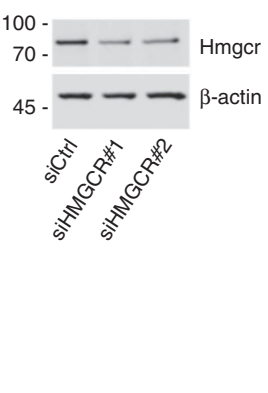

C

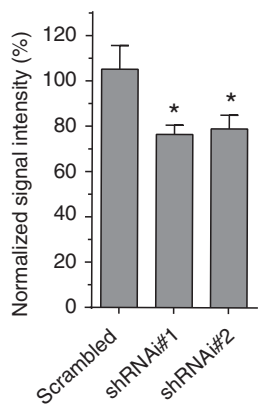

d

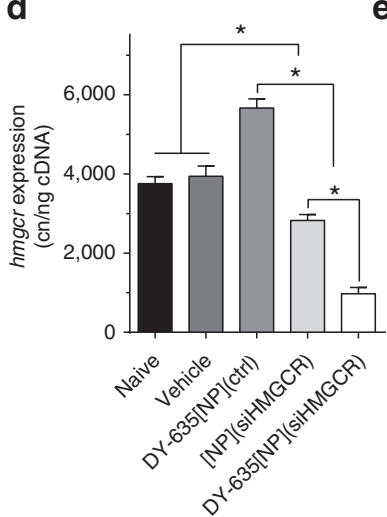

e

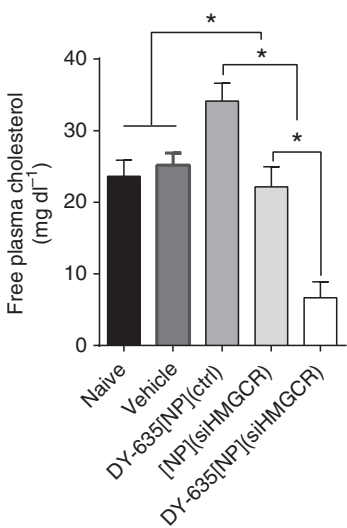

Figure 8 | hmgcr RNAi using DY-635[NP] as carrier. (a) A significant reduction in hmgcr gene-expression was achieved with a maximum at $16 \mathrm{~h}$ after transfection in naive Hepa1-6 cells. In contrast to DY-635[NP](siRNA) an increase in hmgcr was observed after application of the carrier (DY-635[NP](siCtrl)). Mean \pm s.e.m. ${ }^{\star} P<0.05$ compared with respective siCtrl by ANOVA and Tukey post hoc. (b,c) To validate siRNAs, the efficacy of two RNAi sequences (shRNA\#1, shRNA\#2) against hmgcr was analysed in a heterologous expression model using western blot (b), and addressed by determining endogenous HMGCR levels in HepG2 cells using anti-HMGCR immuno-fluorescence analysis (c). A significant reduction in hmgcr protein could be achieved with both RNAis; for further RNAi experiments RNAi sequence\#2 was selected. Mean \pm s.e.m. ${ }^{\star} P<0.05$ versus control by ANOVA and Dunnett's test. (d) In vivo RNAi results in mice injected with [NP](siHMGCR) $(n=9)$ or DY-635[NP] loaded with siHMGCR ( $n=12)$ or a control RNA

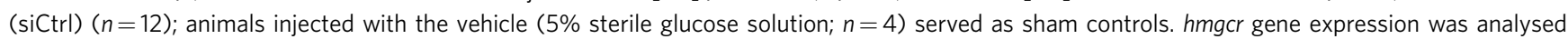
using relative quantification compared with untreated anminals $(n=4)$ in RNA prepared from liver tissue by RT-qPCR. Consistent with the results of the cell culture, DY-635[NP](siCtrl) induced the hmgcr gene expression while [NP](siHMGCR) blunted this effect but failed to lower the $h m g c r$ expression compared with untreated or sham animals. Using similar siHMGCR amounts encapsulated in DY-635[NP](siHMGCR), hmgcr expression was lowered by $75 \%$ compared with untreated animals. Mean \pm s.e.m. ${ }^{\star} P<0.05$ by ANOVA and Tukey test. (e) Altered gene expression of $h m g c r$ was reflected in plasma-cholesterol levels of these animals. Mean \pm s.e.m. by ANOVA and Tukey test.

these transporter systems seems feasible. We could identify organic anion transporter proteins as the transporters responsible for uptake of DY-635. Their critical role in the active targeting of $\mathrm{DY}-635[\mathrm{NP}](-)$ to achieve basolateral uptake into hepatocytes is supported by the observation of cyclosporine A-sensitive uptake by HepaRG cells cultured under flow conditions. Our in vitro and vivo findings suggest a model where the DY-635[NP]s are immobilised at the cell surface of hepatocytes through interaction of DY-635 with OATPs followed by subsequent clathrinmediated endocytosis. Because of their size $(170 \mathrm{~nm})$, DY$635[\mathrm{NP}](-)$ cannot be transported directly by the OATPs ${ }^{40}$ and endocytosis after immobilisation via binding to the uptake transporters on the basolateral membrane is the likely mechanism. Dahlman et al. ${ }^{41}$ have recently reported siRNA encapsulated in NPs that specifically target vascular endothelial cells. The NP appear to be taken up via receptor mediated endocytosis, but this mechanism is distinct from the dye functionalised NPs that we describe. The vascular endothelium is in direct contact with the intravascular space and exhibits effective but not very specific endocytosis. In contrast, hepatocytes are separated from the vascular space and our technology exploits recognition of the transporters that are very specific to the hepatocytes. Thus while the technology of Dahlman et al. appears to be very effective for targeting vascular lining cells, it is not likely to be able to target nonvascular cells.

Likewise, DY-635 labelled PLGA cannot be secreted directly into bile due to its molar mass (MW) of $12 \mathrm{kDa}$ (non-modified polymer) and hydrophobicity. Based on the data in Fig. 5a, showing hepatocyte uptake and excretion of both, nile red cargo and DY-635 as functionalising moiety, degradation by intracellular esterases of PLGA with desorption of DY-635 and subsequent secretion into bile is likely. This is also supported by the observation that the decay of fluorescence followed an exponential function for the free dye, while it was delayed and almost linear for the DY-635[NP]( - )-associated signal. It would also be consistent with a multistep processing within the endosome, with disruption and release of the payload into the cytosol, supported by the cationic polymer PEI ${ }^{42,43}$. Escape of siRNA from the endosome is a critical and rate-limiting step for delivery approaches ${ }^{44}$. Quantification of this delivery for lipidbased NP (LNP) delivery has been previously determined to be in the range of $1-2 \%$ of the applied siRNA ${ }^{44}$. Thus, confirmation of uptake of NPs does not necessarily imply functional delivery of the RNAi therapeutic. Although assessment of the intracellular handling of the cargo is beyond the scope of the present study, we demonstrated a significant inhibition of cholesterol biosynthesis with encapsulation of a siRNA directed against hmgcr into DY635[NP]. Hmgcr was chosen as an exemplary pathway that reflects a key metabolic function of the liver but also based on the observation that DY-635[NP] ( - ) increased plasma cholesterol while toxicology screening otherwise revealed no unwarranted side effects. The observed increase in hmgcr and plasma cholesterol with the empty carrier is a phenomenon likely to be associated with the penetration of the cell membrane NPs leading to an adaptive increase of cholesterol biosynthesis as described for endocytosis ${ }^{45}$ or in response to pore-forming toxins ${ }^{35}$. This side effect may confer protection in the acute setting ${ }^{46}$ but is of concern if repeated administration seems required. The increase in plasma cholesterol could be blunted if siRNA directed against hmgcr was the cargo in non-functionalised NPs, or substantially decreased if the same NPs were functionalised by DY-635 to target hepatocytes as a primary site of cholesterol synthesis.

During degradation of DY-635[NP] (siRNA) and the associated release of the siRNA into the cytosol, DY-635 is presumably desorbed from the polymer and secreted into bile. The zonal distribution of the dye during this process with primary uptake in the pericentral region and subsequent cell-to-cell transport to midzonal and periportal regions of the low-molar mass dye (potentially also an RNAi therapeutic) is likely to explain the 
spreading of the fluorescence signal ostensibly through gap junctions ${ }^{47,48}$.

IVM also confirmed that uptake of DY-635[NP](-) was almost exclusively associated with hepatocytes, primarily in zone 3 of the acinus. No significant accumulation in non-parenchymal cells, most notably Kupffer cells that are primarily sited in zone 1 of the acinus and that take up significant portions of $\mathrm{LNPs}^{49}$, was observed for DY-635[NP] $(-)$. This extends the concept of active targeting of the liver (with a high proportion of phagocytic and immunocompetent cells) to an active and highly specific targeting of liver parenchymal cells, that is, the primary goal for targeted interventions into metabolic pathways.

While fluorescence microscopy is a suitable tool to study these fundamental questions regarding uptake and fate of DY$635[\mathrm{NP}](-)$, the ability to monitor body distribution noninvasively by MSOT provides new opportunities to guide the delivery of RNAi therapeutics. Theranostics, a linguistic blend of diagnostics and therapy, reflects an increasingly important concept in personalising health care. This aims to explain interindividual and population-to-population variabilities in health intervention outcomes using diagnostic tests such that either the type or extent of the intervention can be individualised ${ }^{20}$. Our present approach allows this concept to be applied to the delivery of nano-formulated active drugs, in particular those with significant off-target effects, by predicting the targeted cells and tissues individually before administration of the carrier and cargo.

In summary, the present study demonstrates that uptake receptors can be targeted very efficiently with polymethine dyes. Using DY-635, a dye with known hepatobiliary clearance, we demonstrate that this approach extends the concept of delivery to the liver, for example, with LNPs to exclusive targeting of hepatic parenchymal cells. In addition to exploring a novel, well-defined uptake route the use of dyes to functionalise the NPs also enables their tracking, even non-invasively by MSOT ${ }^{50-52}$. This suggests that organ-selective uptake of a dye can be transferred to NPs to generate theranostic drug carriers allowing fundamental novel options in personalised health care.

\section{Methods}

Animals. Animal studies were conducted in accordance with animal welfare legislation under pathogen-free conditions in the animal facility of the Jena University Hospital or the Helmholtz-Center Munich. During all procedures and imaging methods, animals remained under deep general anaesthesia using Isoflurane and pain-reflexes were assessed to gauge the depth of anaesthesia.

In vivo pharmacokinetics of various polymethine dyes. Catheters were placed in the jugular vein, carotid artery, bladder and bile duct of male Wistar rats (300-400 g body weight (BW)). After i.v. administration of DY-635 (13 pmol per $\mathrm{g}$ BW), DY-630 (13 pmol per g BW), DY-750 (13 pmol per gBW), DY-751 (13 pmol per g BW), DY-731 (13 pmol per g BW), DY-777 (14 pmol per g BW), DY-682 (13 pmol per g BW), DY-704 (17 pmol per g BW), DY-732 (13 pmol per g BW), DY-754 (17 pmol per g BW), DY-678 (14 pmol per g BW), DY-778 (14 pmol per $g$ BW) or $6.5 \mu \mathrm{g}$ DY625-[NP] per g BW, bile was collected every $5 \mathrm{~min}$ and $200 \mu \mathrm{l}$ arterial blood taken at defined times into heparinised tubes. DY-635 fluorescence in bile and plasma was measured in a spectrofluorometer (FluoStar Optima, BMG Labtech). Results are mean \pm s.e.m. of three biological replicates.

Analysis of DY-635 interaction with human hepatic basolateral transporters. HEK293 cells were transfected with OATP1B1 (SLCO1B1, NM_006446), OATP1B3 (SLCO1B3, NM_019844), OCT1 (SLC22A1, NM_003057.2), OAT2 (SLC22A7, NM_006672), NTCP (SLC10A1, NM_003049), NaDC3 (SLC13A3, AF154121) or the empty vector (pcDNA5).Transfected HEK293 cells were seeded at $2 \times 10^{5}$ cells per well and cultured for 3 days. To determine the interaction of DY-635 with different transporters mock- and transporter-transfected cells were incubated with radio-labelled substrates for OATP1B1 $\left(\left[{ }^{3} \mathrm{H}\right]\right.$ estrone-sulfate, $30 \mathrm{nM})$, OATP1B3 $\left(\left[{ }^{3} \mathrm{H}\right]\right.$ sulfobromphthalein, $\left.50 \mathrm{nM}\right)$, OCT1 $\left(\left[{ }^{3} \mathrm{H}\right]\right.$ 1-methyl-4phenylpyridinium iodide, $10 \mu \mathrm{M})$, OAT2 $\left(\left[{ }^{3} \mathrm{H}\right] \mathrm{cGMP}, 10 \mu \mathrm{M}\right)$, NTCP $\left(\left[{ }^{3} \mathrm{H}\right]\right.$ estrone-sulfate, $\left.10 \mu \mathrm{M}\right)$ or $\mathrm{NaDC} 3\left(\left[{ }^{14} \mathrm{C}\right]\right.$ succinate, $\left.10 \mu \mathrm{M}\right)$, in the absence and presence of $10 \mu \mathrm{M}$ DY-635 or an inhibitor for OATP1B1 (rifampicin, $20 \mu \mathrm{M}$ ), OATP1B3 (rifampicin, $20 \mu \mathrm{M}$ ), OCT1 (decynium22, $50 \mu \mathrm{M}$ ), OAT2 (indomethacin, $100 \mu \mathrm{M}$ ), NTCP (cyclosporine A, $50 \mu \mathrm{M}$ ) and NaDC3 (succinate, $100 \mu \mathrm{M}$ )
Hepatocyte isolation. Male FVB/NRj were sacrificed using cervical dislocation, The abdomen was opened and the portal vein catheterised and perfused briefly with pre-warmed Dulbecco's PBS (Sigma Aldrich) containing $0.5 \mathrm{~mm}$ EGTA and $0.1 \%$ Penicillin/Streptomycin at $6.5 \mathrm{ml}$ per min. followed by $4-6 \mathrm{~min}$ perfusion using pre-warmed Dulbecco's PBS containing $0.5 \mathrm{mg}$ collagen per $\mathrm{ml}$ and $1 \mathrm{mM}$ $\mathrm{CaCl}_{2}$ (pH7.4). The digested liver was transferred to Leibovitz-Buffer (Gibco, Life Technologies) and hepatocytes separated andcentrifuged at $50 \mathrm{~g}$ for $5 \mathrm{~min}$ at $4{ }^{\circ} \mathrm{C}$. The pellet was rinsed through a $40 \mu \mathrm{m}$ cell and resuspended with $20 \mathrm{ml}$ ice-cold hepatocyte wash media (Gibco, Life Technologies) containing $0.1 \%$ Penicillin/ Streptomycin. This was repeated seven times to yield highly purified hepatocytes. 80,000 hepatocytes per well were seeded using William's Medium E (10 \% FKS, $0.025 \%$ Insulin (v/v), $0.1 \%$ Glutamaxx (2mM) (Gibco, Life Technologies), $0.1 \%$ Penicillin/Streptomycin). After 6-8h media was changed to remove non-attached hepatocytes.

DY-635 and nanoparticle uptake by primary hepatocytes. Murine primary hepatocytes isolated and cultured on collagen-coated 96-well-plate for 2-3 days were used to measure the uptake of DY-635 (amino-terminated), DY-

$635[\mathrm{NP}](\mathrm{NileRed})$ and [NP](NileRed) in absence and presence of cyclosporine A. Hepatocytes were washed with PBS before a $2 \times$ inhibitor solution containing Krebs-Henseleit buffer (KHB, Biochrom AG), $2 \%$ foetal calf serum and $2 \times$ cyclosporine A was added. This was then diluted 1:1 (v/v) with either $400 \mathrm{nM}$ DY635 (amino-terminated) or $10 \mu \mathrm{g} \mathrm{NP}$ in KHB. After $2 \mathrm{~min}$ (DY-635) or $30 \mathrm{~min}$ (NP) incubation at 37 or $4{ }^{\circ} \mathrm{C}$ media was removed and cells were washed twice with ice-cold PBS containing $50 \mu \mathrm{M}$ cyclosporine A. Cells were lysed in $5 \%$ acetonitrile and $0.1 \%$ Tween-20. Lysates were analysed on a Tecan plate reader (Tecan) (excitation: $488 \pm 09 \mathrm{~nm}$, emission $530 \pm 20 \mathrm{~nm}$ ). A NileRed-standard curve allowed calculation of intracellular NileRed-amounts.A correction factor was calculated from serial dilutions of the NP to correct for different NileRed concentration in $[\mathrm{NP}](\mathrm{NileRed})$ and DY-635[NP](NileRed). Data were normalised to cellular protein (Bradford).

Polymer labeling. Conjugation of acid terminated PLGA (50:50) and aminoterminated DY-635 or DY-704 was performed via EDC chemistry (to label every hundredth polymer chain). $0.95 \mathrm{~g} \mathrm{COOH}$ - terminated PLGA (Sigma-Aldrich, $M_{\mathrm{w}}=7,000$ to $\left.\left.17,000 \mathrm{~g} \mathrm{~mol}^{-1}\right)\right)$ and $0.52 \mathrm{mg}(2.72 \mu \mathrm{mol})$ EDC were reacted in $20 \mathrm{ml}$ dried methylene chloride for $1 \mathrm{~h}$ at room temperature. Subsequently, $1 \mathrm{mg}$ $(1.36 \mu \mathrm{mol}) \mathrm{NH}_{2}$ terminated DY-635 or DY-704 (Dyomics GmbH, Jena, Germany), in $1 \mathrm{ml} \mathrm{DMF}$ was added and stirred at room temperature for $12 \mathrm{~h}$. The solvent was evaporated under reduced pressure and the solid residue dissolved in acetone. The resulting PLGA-DY-635 or PLGA-DY-704 was precipitated (very fast) in cold water to generate a dispersion and this was further purified by dialysis against water using a Spectra/Por 3 dialysis membrane (Spectrumlabs,

$3,500 \mathrm{~g} \mathrm{~mol}^{-1}$ cut-off). Finally, the product was filtered and lyophilised. The calculated labelling efficiency for conjugation was $77 \%$. Size exclusion chromatography was performed on a Shimadzu system (Shimadzu) equipped with a SCL$10 \mathrm{~A}$ system controller, an LC-10AD pump, an RID-10A RI detector, a UVD SPD10AD UV/visible detector and a PSS SDV linear S, $5 \mu \mathrm{m}$ column $(8 \times 300 \mathrm{~mm})$ with chloroform/triethylamine/2-propanol (94:4:2) as eluent at $1 \mathrm{ml} \mathrm{min}^{-1}$, and the column oven was set to $40^{\circ} \mathrm{C}$. A calibration with low polydispersity polystyrene standards (ranging $M_{\mathrm{n}}$ from 380 to $128,000 \mathrm{~g} \mathrm{~mol}^{-1}$ ) was used.

UV/Vis spectroscopy. UV/visible absorption spectra were recorded using a UV/visible spectrometer Specord 250 (Analytik Jena AG, Jena, Germany) in a $1 \times 1 \mathrm{~cm}$ quartz sample cell. The labeling efficiency for conjugation was estimated via $\mathrm{UV} /$ visible spectroscopy using the molar extinction coefficient of the dyes and calculated via equation (1):

$$
\text { Labelling efficiency }=\frac{\text { Experimental labelling degree }}{\text { Theoretical labelling degree }} \times 100
$$

Scanning electron microscopy (SEM). NP suspensions were diluted with deionised water. One droplet was placed on a mica surface and lyophilised for $3 \mathrm{~h}$. Finally, the samples were coated with platinum ( $5 \mathrm{~nm})$, using a BAL-TEC MED020 sputtering device (Bal-Tec AG, Liechtenstein). SEM measurements were performed on a Zeiss (LEO) 1530 Gemini FESEM (Zeiss, Jena, Germany) operating at 8 to $10 \mathrm{kV}$ using the InLens detector.

Dynamic light scattering. Batch DLS was performed on a ZetaSizer Nano ZS (Malvern, Herrenberg, Germany) equipped with a He-Ne laser operating at a wavelength of $633 \mathrm{~nm}$. Counts were detected at an angle of $173^{\circ}$. All measurements were conducted at $25^{\circ} \mathrm{C}$ after an equilibration of $120 \mathrm{~s}$ in triplicate. For analyzing the autocorrelation function, the cumulative analysis and a NNLS algorithm (nonnegative least-squares) were applied. Apparent hydrodynamic radii were calculated using to the Stokes-Einstein Equation (equation 2):

$$
R_{h}=\frac{k T}{6 \pi \eta D}
$$


where, $R_{\mathrm{h}}=$ hydrodynamic radius, $k=$ Boltzmann constant, $T=$ absolute temperature, $\eta=$ viscosity of the sample and $D=$ apparent translational diffusion coefficient.

Electrophoretic light scattering. Electrophoretic light scattering was used to measure the electrokinetic potential (zeta potential). Measurements were performed on a Zetasizer Nano ZS (Malvern Instruments) by applying laser Doppler velocimetry. For each measurement, 10-30 runs were carried out using the slowfield reversal and fast-field reversal modes at $150 \mathrm{~V}$. Each experiment was performed in triplicate at $25^{\circ} \mathrm{C}$. The zeta potential $(\zeta)$ was calculated from the electrophoretic mobility $(\mu)$ according to the Henry Equation (equation 3 ) with $f(k a)=1.5$

$$
\zeta=\frac{3 \eta \mu}{2 \varepsilon f(k a)}
$$

where $\varepsilon=$ dielectric constant and $f(k a)=$ Henry constant.

Nanoparticle formulation. siRNA or indocyanine green (ICG) encapsulated in particles ([NP](siRNA), DY-635[NP](siRNA), [NP](ICG) or DY-635[NP](ICG) were prepared by the double emulsion water/oil/water evaporation, as described ${ }^{53}$. siRNA encapsulation polyplexes of linear PEI (L-PEI) and siRNA (Supplementary Table 2) were prepared in pure water. L-PEI with a degree of polymerisation of 200 was synthesised as previously described ${ }^{54}$. L-PEI and siRNA were diluted in pure water to a stock concentration of $1 \mathrm{mg} \mathrm{ml}^{-1}$. Polyplexes were formed of $8 \mu \mathrm{g}$ siRNA and $9.8 \mu \mathrm{g}$ L-PEI in a total volume of $250 \mu \mathrm{l}$, vortexed briefly, and incubated for $20 \mathrm{~min}$ at room temperature. A total mass of $100 \mathrm{mg}$ PLGA or PLGA-DY-635 were diluted in $5 \mathrm{ml}$ ethylacetate $\left(20 \mathrm{mg} \mathrm{ml}^{-1}\right)$. The polyplex solution was added and the mixture immediately sonicated for 20 s (VibraCell VC505 (500 W); Sonics) with a replaceable $1 / 8$ " tapered microtip, and $30 \%$ amplitude for $20 \mathrm{~s}$. An aqueous solution of $3 \%(\mathrm{w} / \mathrm{v})$ poly(vinyl alcohol) (PVA, $\mathrm{M}_{\mathrm{W}}=67,000 \mathrm{~g} \mathrm{~mol}^{-1}, 86.7$ to $88.7 \mathrm{~mol} \%$ hydrolysed, Sigma Aldrich) (5 ml, PVA diluted in $\mathrm{ddH}_{2} \mathrm{O}$ ) was then added to the first emulsion and sonicated as for the first emulsion. The double emulsion was

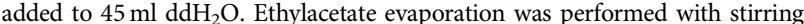
overnight. The particles were washed by centrifugation $(14,000 \mathrm{~g}, 10 \mathrm{~min})$ and twice resuspended in $\mathrm{ddH}_{2} \mathrm{O}$. Particles were lyophilised and stored at $-20{ }^{\circ} \mathrm{C}$ until use. For encapsulation of DY-780 or Nile red, the polymer and dye were diluted in acetone to a final polymer concentration of $7.5 \mathrm{mg} \mathrm{ml}^{-1}$ with $2 \%(\mathrm{w} / \mathrm{w})$ of each dye. The polymer dye solution was transferred to a syringe and automatically injected $\left(13.02 \mathrm{ml} \mathrm{h}^{-1}\right)$ into a three-fold excess of ultra-pure water. To stabilise the formed NPs and to enable lyophilisation $1 \mu \mathrm{g}$ PVA was added to 100 -mg polymer. Finally the NP solution was lyophilised and stored at $-20^{\circ} \mathrm{C}$ until use.

Asymmetric flow field-flow fractionation. AF4 was performed on an AF2000 MT System (Postnova Analytics, Landsberg, Germany) coupled to an UV (PN3211, $260 \mathrm{~nm}$ (Postnova)), RI (PN3150), multi-angle light scattering (MALLS, PN3070, $633 \mathrm{~nm}$ (Postnova)) and DLS (ZetaSizer Nano ZS; Malvern) detector. The eluent was delivered by three different pumps (tip, focus, cross-flow) and the sample injected by autosampler (PN5300) into the channel. The channel has a trapezoidal geometry and an overall area of $31.6 \mathrm{~cm}^{2}$. The nominal height of the spacer was $500 \mu \mathrm{m}$. A regenerated cellulose membrane with a molar mass cut-off of $10 \mathrm{kDa}$ served as accumulation wall. All experiments were carried out at $25^{\circ} \mathrm{C}$ with pure water as eluent. An amount $10 \mu \mathrm{l}$ of sample $\left(1 \mathrm{mg} \mathrm{ml}^{-1}\right)$ was injected with an injection flow rate of $0.2 \mathrm{ml} \mathrm{min}^{-1}$ and a cross-flow rate of $1.2 \mathrm{ml} \mathrm{min}^{-1}$ for $7 \mathrm{~min}$ (detector flow rate $0.5 \mathrm{ml} \mathrm{min}^{-1}$, focus flow rate $1.5 \mathrm{ml} \mathrm{min}^{-1}$ ). After the focusing step, the cross-flow rate was reduced under an exponential gradient (0.4) within $10 \mathrm{~min}$ to $0 \mathrm{ml} \mathrm{min}^{-1}$. The cross-flow was kept constant at $0 \mathrm{ml} \mathrm{min}^{-1}$ for $40 \mathrm{~min}$ to ensure complete elution. All measurements were in triplicate.

Determination of siRNA content. siRNA content of the NPs was determined by determination of total phosphorus according to Chen et al. ${ }^{55}$ Freeze-dried NPs $(10-16 \mathrm{mg})$ and phosphorus standards $\left(0-0.195 \mu \mathrm{mol}, \mathrm{KH}_{2} \mathrm{PO}_{4}\right.$, Sigma-Aldrich) were placed in separate tubes. After adding $0.45 \mathrm{ml}$ of $8.9 \mathrm{~N} \mathrm{H}_{2} \mathrm{SO}_{4}$, tubes were heated at $210^{\circ} \mathrm{C}$ for $25 \mathrm{~min}$. After cooling to room temperature, $150 \mu \mathrm{l} \mathrm{H}_{2} \mathrm{O}_{2}(30$ wt. \%) were added and samples were reheated at $210^{\circ} \mathrm{C}$ for $30 \mathrm{~min}$. After cooling $3.9 \mathrm{ml}$ deionised water and $0.5 \mathrm{ml}$ of ammonium molybdate (VI) tetrahydrate solution ( $2.5 \mathrm{wt}$. \%) were added. After vortexing $(20 \mathrm{~s}), 0.5 \mathrm{ml}$ of L-ascorbic acid solution (10 wt. \%) was added and the tubes vortexed again for $20 \mathrm{~s}$. Tubes were capped and heated for $7 \mathrm{~min}$ at $80^{\circ} \mathrm{C}$. Absorbance of solutions was measured at $820 \mathrm{~nm}$ on a Specord $250 \mathrm{UV} /$ visible-spectrometer (Analytik Jena AG) in a $1 \mathrm{~cm}$ quartz cell. siRNA content was determined based on phosphorous content and number of base pairs in the siRNA.

Multispectral optoacoustic tomography. MSOT was performed as described ${ }^{56}$ Briefly, a commercial MSOT (iThera medical, Munich, Germany) with an array of 256 cylindrically focused transducers was disposed around the mouse and simultaneously collected signals from an optical plane. CD1 or athymic NudeFoxn $1^{\text {nu }}$ mice bearing a MDA-MB-231 human breast cancer xenograft were placed inside the tomograph. Multispectral image stacks were taken from the whole abdomen immediately before and $20 \mathrm{~min}$ after injection of $7 \mu \mathrm{g}$ of either DY635[NP](DY-780), [NP](ICG), DY-635[NP](ICG) or DY-704[NP]( - ) per g BW. DY780 could be only detected by MSOT after release from the NPs. DY-780, DY-704 and ICG signals could be spatially localised upon spectral unmixing of MSOT images and comparison with previously recorded DY-780 spectra. Animals were frozen immediately after the imaging experiment for validation by multispectral epi-illumination cryoslice imaging ${ }^{57}$.

Dynamic cell culture. HUVEC and HepaRG cells were co-cultured using a microfluidic-supported chip. (Supplementary Fig. 2). HepaRG cells were cultured as described ${ }^{58} .2 .7 \times 10^{4}$ cells per $\mathrm{cm}^{2}$ were seeded in William's $\mathrm{E}$ medium supplemented with $10 \%$ foetal bovine serum, $100 \mathrm{U} \mathrm{ml}^{-1}$ penicillin, $100 \mathrm{~g} \mathrm{ml}^{-1}$ streptomycin, $5 \mathrm{U} \mathrm{ml}^{-1}$ insulin and $5 \times 10^{-5} \mathrm{M}$ hydrocortisone-hemisuccinate and cultured for 2 weeks. For HepaRG differentiation, cells were cultured with $2 \%$ dimethylsulfoxide for two more weeks. Differentiated HepaRG cells were seeded at 4,000 cells per $\mathrm{mm}^{2}$ at the bottom of the chip cavities beneath the membrane. 300,000 HUVEC $\left(4,300\right.$ cells per $\left.\mathrm{mm}^{2}\right)$ were seeded above the membrane and cocultured with differentiated HepaRG cells in for $24 \mathrm{~h}$ before the uptake experiments. Cells were perfused with $50 \mu \mathrm{g} \mathrm{ml}^{-1} \mathrm{NPs}$ in Williams E medium. Three independent experiments to assess DY-635[NP] $(-)$ uptake were done for each group (static and dynamic). For each time point three pictures were taken and 200 region of interest (ROI) per picture were analysed. Images in Fig. $6 c$ were corrected by subtracting the mean local background intensity from the mean fluorescence intensity of a neighbouring ROI.

Intravital microscopy. For IVM the jugular vein of male FVB/NRj mice (25 to $30 \mathrm{~g} \mathrm{BW}$ ) was cannulated and the liver exposed via a right lateral abdominal incision 70 pmol DY-635 g $\mathrm{g}^{-1}$ BW (in dimethylsulfoxide $(1 \mathrm{M})$ and diluted to $20 \mu \mathrm{M}$ in $0.9 \% \mathrm{NaCl}$ ) or $7 \mu \mathrm{g}$ DY-635[NP], DY-704[NP], DY-630[NP](NileRed) per $\mathrm{g} \mathrm{BW}\left(1 \mathrm{mg} \mathrm{ml}^{-1}\right.$ in $5 \%$ glucose) were injected i.v. IVM was performed using an inverted epifluorescence microscope (AxioObserverR Z1; Zeiss) as described previously ${ }^{8,30}$. DY-635 or DY-635[NP] ( - ) were visualised at $633 \mathrm{~nm}$ (exposure time $40 \mathrm{~ms}$ ). Co-localisation of DY-635 or DY-635(NP) and liver tissue was performed using 8-bit, grey-value tiff-images and ImageJ with a pseudo colour scale. For Fig. $7 \mathrm{f}$ pictures taken $8 \mathrm{~min}$ after injection of DY-635 and after $28 \mathrm{~min}$ (8 min after DY-635[NP] $(-)$ injection) were overlaid and colocalised pixels counted. This number was then corrected for the amount of colocalised pixel that might persist from the injection of free dye DY-635 using the following formula for DY-635 relative fluorescence decrease: $\mathrm{FI}(\mathrm{DY}-635)[\%]=71.6^{- \text {time } / 12.3}+$ $151,9^{- \text {time/3.7 }}$ (Fig. 7 c)

In vivo confocal laser scanning microscopy. For in vivo confocal laser scanning microscopy a tail-vein catheter (30G) was placed in male FVB/NRj mice (25-30 g $\mathrm{BW})$. The liver or/ and kidney was exposed and placed on a cover-slip. Images were acquired using a LSM-780 (Zeiss) using an air corrected $\times 40$ plan-apochromatic objective (NA: 0.95). Lasers and filters used are summarised in Supplementary Table 3 NPs or free dyes were injected in similar concentration as described above via the tail vein-catheter.

HMGCR-RNAi validation in vitro. Hepa1-6 cells were cultured in DMEM with $10 \% \mathrm{FBS}$ and penicillin-streptomycin. $8 \times 10^{5}$ cells per well were seeded in 12 -well plates $24 \mathrm{~h}$ prior to transfection. Lyophilised DY-635[NP](siHMGCR) were dissolved in OptiMem (Life technologies) to $500 \mu \mathrm{g} \mathrm{ml}^{-1}$. Cells were washed twice in PBS and incubated with $1 \mathrm{ml}$ DY-635[NP](siHMGCR). After $4 \mathrm{~h}$ media were changed to normal growth media for a further 12 or $20 \mathrm{~h}$ and $\mathrm{hmgcr}$ steady state transcript levels assessed by reverse transcriptase-quantitative PCR (RT-qPCR) (Supplementary Table 4). To measure HMGCR-protein knockdown efficacy, HEK293 cells were transfected with pRNAT H1.1 vectors encoding the hmgcrshRNA used for NP encapsulations (Supplementary Table 3) as well as mouse GFP-HMGCR (419-887) instead of GFP (for plasmid and cloning information see Supplementary Fig. 3). After $24 \mathrm{~h}$ cells were lysed and protein levels analysed via anti-GFP immunoblotting 59,60

To confirm the heterologous $h m g c r$ RNAi results by immunofluorescence analyses of endogenous HMGCR, anti-human HMGCR antibodies (Santa Cruz) were tested for recognition of purified recombinant HMGCR(aa419-887) fusion proteins of overexpressed murine GFP-HMGCR(aa419-887) and of endogenous HMGCR. For RNAi validations in HepG2-cells, cells transfected with GFPcoexpressing RNAi vectors were processed for fluorescence analysis using a Zeiss CellObserver with an apotome. $16 \mathrm{~h}$ after transfection Z-stacks were accumulated and integrated intensities of SUM-projections of the anti-HMGCR signal were measured in ImageJ. Two independent cellular assays with $n>16$ cells were conducted.

The effects of siHMGCR\#2 on gene expression were assessed in $8 \times 10^{5}$ Hepal6 cells incubated for $24 \mathrm{~h}$ with $2 \mathrm{ml}$ full growth media (DMEM/F12, $10 \% \mathrm{FCS}, 1 \%$ penicillin/streptomycin) at $37^{\circ} \mathrm{C}, 5 \% \mathrm{CO}_{2}$. Directly before transfection lyophilised DY-635[NP] (siHMGCR) or DY-635[NP(siCtrl) were dissolved in OptiMEM (Life Technologies) to a final concentration of 100,200 or $500 \mu \mathrm{g}$ NP per ml. Growth Media were removed and the cells washed twice with PBS. $1 \mathrm{ml}$ of the NP-solutions was added to the cells. Four hours later the NP was removed and full growth media 
added. Cells were harvested after an additional 14 or $20 \mathrm{~h}$ for RT-qPCR. See Supplementary Table 2 for primer-pairs. Results of three independent experiments with three technical replicates were used for in vitro gene expression analysis. Data are mean \pm s.e.m.

In vivo RNAi. A venous access port was placed in male FCB/NRj mice at 5 weeks. The mice were treated with antibiotics for 7 days to prevent infection. Fourteen days after the operation lyophilised [NP](siHMGCR), DY-635[NP](siHMGCR) or DY-635[NP] (siCtrl) were dissolved in 5\% glucose to a concentration of $1 \mathrm{mg}$ NP per ml. $7 \mu \mathrm{g} \mathrm{NP}(7 \mu \mathrm{l})$ per g BW was administered twice i.v. within $24 \mathrm{~h}$. The mice were sacrificed $16 \mathrm{~h}$ after the second injection and blood collected into heparinised tubes. Plasma and organs were frozen for subsequent analysis. Metabolites were analysed from randomised, blinded samples of plasma (DRI-CHEM 3500i; Fujifilm). Gene expression was analysed in randomised tissue homogenates. $n=4-9$ (individual number are in the legend for Fig. 8).

Statistics. Data were tested for normal distribution and analysed by appropriate analysis of variance (ANOVA) models as described in figure legends. Post-hoc analysis was performed using Dunnett's test when comparing multiple groups with a single control or Tukey test when performing post hoc pair-wise analysis.

\section{References}

1. de Fougerolles, A., Vornlocher, H. P., Maraganore, J. \& Lieberman, J. Interfering with disease: a progress report on siRNA-based therapeutics. Nat. Rev. 6, 443-453 (2007).

2. Kole, R., Krainer, A. R. \& Altman, S. RNA therapeutics: beyond RNA interference and antisense oligonucleotides. Nat. Rev. 11, 125-140 (2012).

3. Davidson, B. L. \& McCray, Jr. P. B. Current prospects for RNA interferencebased therapies. Nat. Rev. 12, 329-340 (2011).

4. Dudek, H. et al. Knockdown of $\beta$-catenin with dicer-substrate siRNA reduces liver tumor BUrden in vivo. Mol. Ther. 22, 92-101 (2014).

5. Shim, M. S. \& Kwon, Y. J. Efficient and targeted delivery of siRNA in vivo. FEBS J. 277, 4814-4827 (2010).

6. Hong, B. J., Chipre, A. J. \& Nguyen, S. T. Acid-Degradable Polymer-Caged Lipoplex (PCL) Platform for siRNA Delivery: Facile Cellular Triggered Release of siRNA. J. Am. Chem. Soc. 135, 17655-17658 (2013).

7. David, S., Pitard, B., Benoit, J. P. \& Passirani, C. Non-viral nanosystems for systemic siRNA delivery. Pharmacol. Res. 62, 100-114 (2010).

8. Yu, B. et al. Targeted nanoparticle delivery overcomes off-target immunostimulatory effects of oligonucleotides and improves therapeutic efficacy in chronic lymphocytic leukemia. Blood 121, 136-147 (2013).

9. Recknagel, P. et al. Liver dysfunction and phosphatidylinositol-3-kinase signalling in early sepsis: experimental studies in rodent models of peritonitis. PLoS Med. 9, e1001338 (2012).

10. Wang, J., Lu, Z., Wientjes, M. G. \& Au, J. L. Delivery of siRNA therapeutics: barriers and carriers. AAPS J. 12, 492-503 (2010).

11. Whitehead, K. A., Langer, R. \& Anderson, D. G. Knocking down barriers: advances in siRNA delivery. Nat. Rev. 8, 129-138 (2009).

12. Qasim, W. \& Thrasher, A. J. RISC control for gene therapy. Nat. Biotechnol. 24, 661-662 (2006).

13. Buyens, K. et al. Liposome based systems for systemic siRNA delivery: stability in blood sets the requirements for optimal carrier design. J. Control Release 158, 362-370 (2012).

14. Islam, M. A. et al. Major degradable polycations as carriers for DNA and siRNA. J. Control Release. doi:10.1016/J.Jcontel.2014.05.055 (in the press).

15. Nishina, K. et al. Efficient in vivo delivery of siRNA to the liver by conjugation of alpha-tocopherol. Mol. Ther. 16, 734-740 (2008).

16. Soutschek, J. et al. Therapeutic silencing of an endogenous gene by systemic administration of modified siRNAs. Nature 432, 173-178 (2004).

17. Mingozzi, F. \& High, K. A. Therapeutic in vivo gene transfer for genetic disease using AAV: progress and challenges. Nat. Rev. 12, 341-355 (2011).

18. Wagner, E. Polymers for siRNA delivery: inspired by viruses to be targeted, dynamic, and precise. Acc. Chem. Res. 45, 1005-1013 (2012).

19. Kay, M. A. State-of-the-art gene-based therapies: the road ahead. Nat. Rev. 12, 316-328 (2011).

20. Ozdemir, V. et al. Shifting emphasis from pharmacogenomics to theragnostics. Nat. Biotechnol. 24, 942-946 (2006).

21. Pan, D. P. J. Theranostic Nanomedicine with Functional Nanoarchitecture Mol. Pharmaceut. 10, 781-782 (2013).

22. Quaglia, A. et al. Liver after hepatocyte transplantation for liver-based metabolic disorders in children. Cell Transplant. 17, 1403-1414 (2008).

23. Dhawan, A., Mitry, R. R. \& Hughes, R. D. Hepatocyte transplantation for liver-based metabolic disorders. J. Inherit. Metab. Dis. 29, 431-435 (2006).

24. Schroit, A. J., Madsen, J. \& Nayar, R. Liposome-cell interactions: in vitro discrimination of uptake mechanism and in vivo targeting strategies to mononuclear phagocytes. Chem. Phys. Lipids 40, 373-393 (1986).
25. Sato, A., Takagi, M., Shimamoto, A., Kawakami, S. \& Hashida, M. Small interfering RNA delivery to the liver by intravenous administration of galactosylated cationic liposomes in mice. Biomaterials 28, 1434-1442 (2007).

26. Akinc, A. et al. Development of lipidoid-siRNA formulations for systemic delivery to the liver. Mol. Ther. 17, 872-879 (2009).

27. Roth, M., Araya, J. J., Timmermann, B. N. \& Hagenbuch, B. Isolation of modulators of the liver-specific organic anion-transporting polypeptides (OATPs) 1B1 and 1B3 from Rollinia emarginata Schlecht (Annonaceae). J. Pharmacol. Exp. Ther. 339, 624-632 (2011).

28. Stieger, B. The role of the sodium-taurocholate cotransporting polypeptide (NTCP) and of the bile salt export pump (BSEP) in physiology and pathophysiology of bile formation. Handbook of experimental pharmacology 201, 205-259 (2011).

29. Okamoto, E., Kyo, A., Yamanaka, N., Tanaka, N. \& Kuwata, K. Prediction of the safe limits of hepatectomy by combined volumetric and functional measurements in patients with impaired hepatic function. Surgery 95, 586-592 (1984).

30. Cherrick, G. R., Stein, S. W., Leevy, C. M. \& Davidson, C. S. Indocyanine green: observations on its physical properties, plasma decay, and hepatic extraction. $J$. Clin. Invest. 39, 592-600 (1960).

31. Leevy, C. M., Mendenhall, C. L., Lesko, W. \& Howard, M. M. Estimation of hepatic blood flow with indocyanine green. J. Clin. Invest. 41, 1169-1179 (1962).

32. Recknagel, P., Claus, R. A., Neugebauer, U., Bauer, M. \& Gonnert, F. A. In vivo imaging of hepatic excretory function in the rat by fluorescence microscopy. J. Biophoton 5, 571-581 (2012).

33. Huang, S-M., Temple, R., Throckmorton, D. C. \& Lesko, L. J. Drug Interaction Studies: Study Design, Data Analysis, and Implications for Dosing and LabelingPublic Policy. Clin. Pharmacol. Ther. 81, 298-304 (2007).

34. Burchard, W. Solution properties of branched macromolecules. Adv. Polym. Sci. 143, 113-194 (1999)

35. Gurcel, L., Abrami, L., Girardin, S., Tschopp, J. \& van der Goot, F. G. Caspase-1 activation of lipid metabolic pathways in response to bacterial pore-forming toxins promotes cell survival. Cell 126, 1135-1145 (2006).

36. Chumakova, O. V. et al. Composition of PLGA and PEI/DNA nanoparticles improves ultrasound-mediated gene delivery in solid tumors in vivo. Cancer Lett. 261, 215-225 (2008).

37. Liang, G. F. et al. PLGA-based gene delivering nanoparticle enhance suppression effect of miRNA in HePG2 cells. Nanoscale Res. Lett. 6, 447 (2011)

38. Patil, Y. \& Panyam, J. Polymeric nanoparticles for siRNA delivery and gene silencing. Int. J. Pharm. 367, 195-203 (2009).

39. Su, W. P., Cheng, F. Y., Shieh, D. B., Yeh, C. S. \& Su, W. C. PLGA nanoparticles codeliver paclitaxel and Stat3 siRNA to overcome cellular resistance in lung cancer cells. Int. J. Nanomed. 7, 4269-4283 (2012).

40. Karlgren, M. et al. In vitro and in silico strategies to identify OATP1B1 inhibitors and predict clinical drug-drug interactions. Pharm. Res. 29, 411-426 (2012).

41. Dahlman, J. E. et al. In vivo endothelial siRNA delivery using polymeric nanoparticles with low molecular weight. Nat. Biotechnol. 9, 648-655 (2014).

42. Cartiera, M. S., Johnson, K. M., Rajendran, V., Caplan, M. J. \& Saltzman, W. M. The uptake and intracellular fate of PLGA nanoparticles in epithelial cells. Biomaterials 30, 2790-2798 (2009).

43. Panyam, J., Zhou, W. Z., Prabha, S., Sahoo, S. K. \& Labhasetwar, V. Rapid endo-lysosomal escape of poly(DL-lactide-co-glycolide) nanoparticles: implications for drug and gene delivery. FASEB J. 16, 1217-1226 (2002).

44. Gilleron, J. et al. Image-based analysis of lipid nanoparticle-mediated siRNA delivery, intracellular trafficking and endosomal escape. Nat. Biotechnol. 31, 638-646 (2013).

45. Puri, V. et al. Cholesterol modulates membrane traffic along the endocytic pathway in sphingolipid-storage diseases. Nat. Cell Biol. 1, 386-388 (1999).

46. Weber, M. et al. Hepatic induction of cholesterol biosynthesis reflects a remote adaptive response to pneumococcal pneumonia. FASEB J. 26, 2424-2436 (2012).

47. Neijssen, J. et al. Cross-presentation by intercellular peptide transfer through gap junctions. Nature 434, 83-88 (2005).

48. De Maio, A., Gingalewski, C., Theodorakis, N. G. \& Clemens, M. G. Interruption of hepatic gap junctional communication in the rat during inflammation induced by bacterial lipopolysaccharide. Shock 14, 53-59 (2000)

49. Kamps, J. A., Morselt, H. W., Swart, P. J., Meijer, D. K. \& Scherphof, G. L. Massive targeting of liposomes, surface-modified with anionized albumins, to hepatic endothelial cells. Proc. Natl Acad. Sci. USA 94, 11681-11685 (1997).

50. Su, R., Ermilov, S. A., Liopo, A. V. \& Oraevsky, A. A. Three-dimensional optoacoustic imaging as a new noninvasive technique to study long-term biodistribution of optical contrast agents in small animal models. J. Biomed. Opt. 17, 101506 (2012).

51. Tsyboulski, D. A. et al. Enabling in vivo measurements of nanoparticle concentrations with three-dimensional optoacoustic tomography. J. Biophoton 7, 581-588 (2014). 
52. Wang, L. V. \& Hu, S. Photoacoustic tomography: in vivo imaging from organelles to organs. Science 335, 1458-1462 (2012).

53. Cohen, J. L. et al. Acetal-modified dextran particles for siRNA delivery. Bioconj. Chem. 22, 1056-1065 (2011).

54. Rinkenauer, A. C. et al. Parallel high-throughput screening of polymer vectors for nonviral gene delivery: evaluation of structure-property relationships of transfection. ACS Comb. Sci. 15, 475-482 (2013).

55. Chen, P. S., Toribara, T. Y. \& Warner, H. Microdetermination of phosphorus. Anal. Chem. 28, 1756-1758 (1956).

56. Razansky, D., Buehler, A. \& Ntziachristos, V. Volumetric real-time multispectral optoacoustic tomography of biomarkers. Nat. Protocol. 6 1121-1129 (2011).

57. Sarantopoulos, A., Themelis, G. \& Ntziachristos, V. Imaging the biodistribution of fluorescent probes using multispectral epi-illumination cryoslicing imaging. Mol. Imaging Biol. 13, 874-885 (2011).

58. Gripon, P. et al. Infection of a human hepatoma cell line by hepatitis B virus. Proc. Natl Acad. Sci. USA 99, 15655-15660 (2002)

59. Schwintzer, L. et al. The functions of the actin nucleator Cobl in cellular morphogenesis critically depend on syndapin I. EMBO J. 30, 3147-3159 (2011).

60. Dharmalingam, E. et al. F-BAR proteins of the syndapin family shape the plasma membrane and are crucial for neuromorphogenesis. J. Neurosci. 29, 13315-13327 (2009)

\section{Acknowledgements}

Grant support by the German Federal Ministry of Education and Research (BMBF) within the framework of the PROGRESS consortium (01KI07111) is gratefully acknowledged, as well as the Carl Zeiss Foundation and the Thuringian Ministry for Education, Science and Culture (TMBWK; \#B514-09051, NanoConSens). Our research was further supported by Dyomics GmbH, Jena, Germany who kindly provided their dyes for polymer-synthesis and screening. We thank C. Fritzsche for preparation of some of the NPs and performance of cytotoxicological and hemocompatibility studies, S. Hoeppener for performance of cryoTEM measurements and Steffi Stumpf for assistance in the SEM investigations as well as A. Keib and U. Vetterling for support during animal experiments and sample-preparation. We thank Stephanie Schubert for help in the particle preparation and helpful comments and J. Hüttemeister for help with RNAi validations.

\section{Author contributions}

Adrian T. Press: Planning, performing and analyzing experiments (Figs 1a-d, 2b,h, 5, 6, 7, 8a,d,e), Planning experiments (Figs 3, 4a, 5, 6), statistical analysis of the data, preparation of graphs and figures; wrote parts of the manuscript. Anja Traeger: Planning and supervising the particle formation and characterisation; wrote parts of the manuscript. Alexander Mosig: Planning, performing and analyzing experiments
(Fig. 5a-d). Christian Pietsch: Planning, performing and analyzing the polymer-synthesi and characterisation (Supplementary Fig. 1a,c, 2d,f). Michael Wagner: Planning and performing the characterisation of the nanoparticles and loading efficiency (Fig. 2c, Supplementary Fig. 1). Mark Clemens: Planning and analyzing in vivo LSM experiment (Fig. 5b), planning and analyzing primary hepatocyte experiments (Figs $1 \mathrm{~d}$ and $2 \mathrm{~h}$ ), planning statistical analysis, writing portions of the manuscript. Nayla Jbeily: Mainly contributed to the performance of intravital microscopy experiments. Nicole Koch: Planning, performing and analyzing experiments for RNAi and anti-HMGCR antibody (Fig. 7b,c and Supplementary Fig. 3). Michael Gottschaldt: Scientific supervision of nanoparticle analysis, supervised and designed nanoparticle characterisation. Volodymyr Ermolayev and Nicolas Bézière: Performed MSOT experiments. Vasilis Ntziachristos: Planned, designed and supervised MSOT analyses. Jürgen Popp: Suggested use of MSOT to image nanoparticles; planned and designed biophotonic analyses. Michael M. Kessels: Planning and scientific supervision of $h m g \mathrm{cr}$ cloning and immunodetections, wrote parts of the manuscript. Britta Qualmann: Planning and scientific supervision of $\mathrm{hmgcr}$ cloning and immunodetections, wrote parts of the manuscript. Ulrich S. Schubert: Design of study (chemistry), planning and supervision of nanoparticle design, wrote the manuscript. Michael Bauer: Design of study (life sciences), planning of functionalised nanoparticles, wrote the manuscript.

\section{Additional information}

Supplementary Information accompanies this paper at http://www.nature.com/ naturecommunications

Competing financial interests: A.T., U.S.S. and M.B. are co-founders of SmartDyeLivery $\mathrm{GmbH}$, a university spin-off company engaged in the development of a platform for nanoformulated drugs to restore critical cellular signalling functions. The IP based upon the present data will be transferred from the University Hospital Jena and the FriedrichSchiller University Jena to SmartDyeLivery GmbH

Reprints and permission information is available online at http://www.nature.com/ reprintsandpermissions/

How to cite this article: Press, A. T. et al. Cell type-specific delivery of short interfering RNAs by dye-functionalised theranostic nanoparticles. Nat. Commun. 5:5565 doi: $10.1038 /$ ncomms6565 (2014)

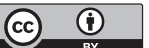

This work is licensed under a Creative Commons Attribution 4.0 International License. The images or other third party material in this article are included in the article's Creative Commons license, unless indicated otherwise in the credit line; if the material is not included under the Creative Commons license, users will need to obtain permission from the license holder to reproduce the material. To view a copy of this license, visit http://creativecommons.org/licenses/by/4.0/ 